\title{
Continuum Regularization of Quantum Field Theory *
}

\author{
Zvi Bern
}

LBL -22303

Ph.D. Thesis

DE87 002549

\author{
Lawrence Berkeley Laboratory \\ and \\ Department of Physics \\ University of California \\ Berkeley, California $9 / 720$
}

\section{DISCLAIMER}

\begin{abstract}
This report was prepared as an account of work sponsored by an agency of the United States Government. Neither the United States Government nor any agency thereof, nor any of their employees, makes any warranty, express or implied, or assumes any legal liability or responsibility for the accuracy, completeness, or usefulness of any information, apparatus, product, or process disclosed, or represents that its use would not infringe privately owned rights. Reference herein to any specific commercial product, process, or service by trade name, trademark, manufacturer, or otherwise does not necessarily constitute or imply its endorsement, recommendation, or favoring by the United States Government or any agency thereof. The views and opinions of authors expressed herein do not necessarily state or reflect those of the United States Government or any agency thereof.
\end{abstract}

\footnotetext{
- This work was supported by the Director, Office of Energy Research, Office of High Energy and Nuclear Physics, Divinion of High Energy Physica of the U.S. Department of Energy under Contract DE-AC0376 SF00098.
} 


\section{Abstract}

In this thesis I discuss possible nonperturbative continuum regularization schemies for quantum field theory which are based upon the Langevin equation of Parisi and Wu.

Breit, Gupta and Zaks made the first proposal for new gauge invariant nonperturbative regularization. The scheme is based on smearing in the "fifth-time" of the Langevin equation. An analysis of their stochastic regularization scheme for the case of scalar electrodynamics with the standard covariant gauge fixing is given. Their scheme is shown to preserve the masslessness of the photon and the tensor structure of the photon vacuum polarization at the one-loop level.

Although stochastic regularization is viable in one-loop electrodynamics, two difficulties arise which, in general, ruins the the scheme. One problem is that the superficial quadratic divergences force a bottomless action for the noise. Another difficulty is that stochastic regularization by fifth-time smearing is incompatible with Zwanziger's gauge fixing, which is the only known nonperturbative covariant gauge fixing for nonabelian gauge theories.

Finally, a successful covariant derivative scheme is discussed which avoids the difficulties encountered with the earlier stochastic regularization by fifthtime smearing. For QCD the regularized formulation is manifestly Lorentz invariant, gauge invariant, ghost free and finite to all orders. A vanishing 
gluon mass is explicitly verinied at one loop. The method is designed to respect relevant symmetries, and is expected to provide suitable regularization for any theory of interest. Hopefully, the scheme will lend itself to nonperturbative analysis. 


\section{Acknowledgements}

I wish to thank Professor Martin B. Halpern for his invaluable advice, teaching and encouragement throughout my graduate studies.

I should like to thank all the members of the theoretical physics group at Berkeley for their friendship and hospitality. In particular, I wish to thank Professors Orlando Alvarez, Mary Kay Gaillard, Allan Kaufman, Mahiko Suruki, and Bruno Zumino for the excellent courses they taught.

The summer that I spent with Allan Kaufman's plasma group was a wonderful experience. I learned much from his unique way of doing physics.

I have also benefitted greatly from discussions and collaborations with with Hue Sun Chan, Nick Kalivas and Lorenzo Sadun. My interests have been widened by conversations with my fellow theory graduate students at LBL and Berkeley. I thank Uwe Albertin, Oren Cheyette, Mitch Golden, Dae Sung Hwang, Randy Ingermanson, Yeong-Chuan Kao, Shobhit Mahajan, Juan Mañes, Adlaj Smith, Eric Sowa, Launey Thomas, Matt Visser and Jonathan Yamron. I also thank Ken Rimey for help with Macsyma.

I thank Mary Gorman, Betty Moura and Luanne Neumann for their friendliness and belp, making LBL so much more pleasant. Additionally, I thank James Lee Lapugh, the LBL security guard, for his friendliness.

I want to thank my parents for all they did to help me get to where I am now. 
Finally, I thank Debby Wagger for her friendship and love as well as for introducing me to hot tubs.

This work was supported by the Director, Office of Energy Research, Office of High Energy and Nuclear Physics, Division of High Energy Physics of the US Department of Energy under Contract DE-AC0376SF00098 and National Science Foundation under Research Grant No. 85-15857. 


\section{Contents}

Acknowledgements

I Introduction

II Overview of Stochastic Quantization

6

III Stochastic Regularization of Scalar Electrodynamics

1 Stochastic Regularization $\ldots \ldots \ldots \ldots \ldots \ldots$

2 Stochastic Regularization of Perturbative Scalar Electrodynamics . . . . . . . . . . . . 17

3 Diagrammatic Proof of the Ward Identities to All Orders . . 29

4 Conclusions and Comments . . . . . . . . . . . 33

IV Incompatibility of Stochastic Regularization and Zwanziger's Gauge Fixing

1 Stochastic Regularization with Zwanziger's Gauge Fixing . . 34

2 Incompatibility in Yang-Mills $\ldots \ldots \ldots \ldots \ldots \ldots$

3 Incompatibility in Scalar Electrodynamics . . . . . . . . 43

v Covariant Derivative Regularization

1 Regularized Langevin System for Gauge Theory . . . . . . . . 44 
2 Vanishing Gluon Mass $\ldots \ldots \ldots \ldots \ldots \ldots$

VI Conclusions

VII References

61

VII Figure Captions

65

DX Figures

66 


\section{Chapter I: Introduction}

The dream of nonperturbative covariant-derivative regularization ior sontinuum gauge theory came essentially to a stop in 1972, when Lee and ZinnJustin [1] observed that the regularization of the propagators was cancelled by high-derivative vertices at the one-loop level. Slavnov $[2,3]$ has since proposed a hybrid action scheme, superposing an intricate special treatment of ono-loop diagrams, in the Faddeev-Popov approach [4] to covariant gauges. Until the Gribov ambiguity [5] in this approach is resolved, however, this scheme must be considered as a perturbative prescription. At least two geometric $[6,7]$ approaches to the problem have also been proposed, but, in both cases, we are unaware of detailed perturbative analysis.

In 1981, Parisi and Wu [8] introduced stochastic quantization, an extension to quantum field theory of earlier work in the theory of stochastic processes [9]. The program reduces quantum field theory to a Gaussian stochantic process called the Langevin equation, which usually runs in an auxiliary "fifth-time". At first sight, stochastic quantization appears to be no more than an anusing alternative to conventional Hamiltonian and action formulations. In fact, however, the approach has given birth to a number of new ideas which are by no means obvious in the conventional formulations. Among these we mention Zwanziger's gauge-fixing $[10,11,12]$, large $N$ quenching $[13,14]$, large $N$ master fields [14], stochastic stabilization [15], 
stochastic regularization $[16,17,18,19,20]$, the $Q C D$, maps which run in ordinary time [21], and numerical applications of the Langevin equation in lattice gauge theory [22].

Stochastic regularization by fifth-time smearing as proposed by Breit, Gupta and Zaka [17] was the first proposa] for new gauge-invariant regularization, and is based on the ideas inherent in stochastic quantization. Hown ever, the conclusion reached by these authors was that the applicability of their atochastic regularization to perturbative computations is problematic. The clairn was, that although the oymmetries of the theory are preserved, the naive conservation laws are not preserved, so that stochastic regularization may not be a satisfactory scheme. However, the relevance of this fact to regularization and renormalization is not clear. For example, Slavnov's hybrid higher covariant derivative method ruins the conservation of the naive Noethy currents, but is certainly a good perturbative regularization scheme for gauge theories [3].

Furthermore, Breit, Gupta and Zaks attempted to apply their idea to fermionic QED which is unfortunately elouded by technical difficulties associated with the noriequilibration of the naive Langevin equation [23]. As first pointed out by Ishikawa [24], and later analyzed in detail by Kalfuss and Meissner [25], the breakdown of gauge invariance encountered by Breit, Gupta and Zaks can be traced to their use of a Langevin equation which was not manifestly gauge invariant. Ishikawa has also proposed a Langevin 
equation for fermions which avoids these problems [24].

To avoid the technicalities associated with the stochastic quantization of fermi fields, we chose to analyze the Briet, Gupta, and Zaks stochastic regularization scheme in scalar electrodynamics (SED), a discussion of which is given in Chapter III as well as Ref. [19]. In contrast to their results for fermions, for SED the scheme preserves the masslessness of the photon and the tensor structure of the photon vacuum polarization at the one-loop level. Furthermore, the scalar wavefuncticn renormalization, $Z_{2}$, is shown to be equal to the one-photon vertex renormalization, $Z_{1}$, to all orders of the stochastically regularized theory.

Although the original difficulties encountered by Briet, Gupta and Zaks have been eircumvented, further difficulties arise which are discussed in Chapters III and IV as well as in Ref. [26]. One problem is that the superficial quadratic divergences of gauge theories force a bottomless action for the noise which ruins the nonperturbative quality of the scheme. Another difficulty is that fifth-time smearing is incompatible with Zwanziger's gauge fixing [10], which is the only known nonperturbative [11] covariant gauge bxing for nonabelian gauge theories. Furthermore, the resulting nonMarkovian evolution loses much of the formal machinery of the unregulated Markov process, making the analysis of the system quite dificult.

To avoid the problems associated with stochastic fegularization by 6fth- 
time amearing, we have developed [27] a covariant derivative regularization scheme which is also based on stochastic quantization. We retain in distinction to Ref. [17], all the technical advantages of a Markoy process, including closed form equilibrium equations, which are the Schwinger-Dyson equations, and Zwanziger's gauge fixing if desired. The resulting regularized theory is manifestly Lorentz invariant, gauge invariant, ghost-free and ultraviolet finite to all orders. We are hopeful that the formulation will lend itself to nonperturbative analysis. ${ }^{1}$

In chapter $\mathrm{V}$, the Langevin formulation of the covariant derivative regularization scheme is set up for the case of Yang-Mills. Also, a one-loop computation of the vaninhing gluon mass is included as an explicit verification of gauge invariance. Further details of the scheme can be found in the series of papers Refo. $[29,30,31,32]$.

The first paper I [29] in the series is on scalar feld theory and is designed partiy as a pedagogical vehicle to introduce, in the simplest possible context, the relevant regularized Langevin and regularized Schwinger-Dyson techniquea. The scalar context is also the simplest in which to study the curiow feature that the scheme is not an action regularization, a fact which is crucial to its succesa in regulating theories with local symmetries. A renormalization program is checked through one loop, including a computation of

\footnotetext{
${ }^{1}$ After the completion of this work, a nonperturbative analysis appeared $|28|$ uaing the ecalar prototype regulator described in Ref. [29|.
} 
the $\beta$-function in $\phi_{B}^{3}$ and a check of unitarity of the renormalized theory.

The second paper II [30] dicusses the case of gauge theories in detail. Among the topies covered are regularized Langevin equations and diagrams for Yang-Mills, regularized Schwinger-Dyson equations and diagrams, finiteness of the Green functions to all orders and regularized functional Laplacians.

The third paper III [31] discusses the one-loop Yang-Mills $\beta$-function in the scheme, demonstrating that the usual results are obtained. Finally, the fourth paper in the series IV [32] discusses some further aspects of renormalization.

Two additional papers describe the case when an exponential (heat kemel [33]) is used as the regulator function [34] and the case when fermions are introduced into the system [35]. 


\section{Chapter II. Overview of Stochastic Quantization}

Stochastic quantization is based upon some well known ideas in nonequilibrium statistical mechanics [9]. For simplicity, at first, the stochastic quantization of a single scalar feld, $\phi$, with action, $S[\phi]$, will be considered. The usual starting point of stochastic quantization [8] is the Langevin equation,

$$
\frac{\partial \phi(x, t)}{\partial t}=-\frac{\delta S[\phi]}{\delta \phi(x, t)}+\eta(x, t)
$$

in which $t$ is a fictitious fifth-time variable, not to be confused with physical time and $x$ represents the four physical space-time dimensions. Here, $\eta$ is a five dimensional random feld with Gaussian probability distribution,

$$
\left\langleF \left[\phi(\eta)|\rangle_{\eta} \equiv \frac{\int D_{\eta} F\left[\phi(\eta) \mid \exp \left(-\frac{1}{4} \int \eta^{2}(x, t) d^{4} x d t\right)\right.}{\int D \eta \exp \left(-\frac{1}{4} \int \eta^{2}(x, t) d^{4} x d t\right)} .\right.\right.
$$

By evaluating the generating functional, $\left\langle\exp \left(\int J_{\eta} d^{4} x d t\right)\right\rangle_{\eta}$, all the n-point $\eta$ correlation function can easily be calculated. After a simple calculation the two point correlation is found to be

$$
\left\langle\eta(x, t) \eta\left(x^{\prime}, t^{\prime}\right)\right\rangle_{\eta}=2 \delta^{4}\left(x-x^{\prime}\right) \delta\left(t-t^{\prime}\right) .
$$

while all other connected $\eta$ correlations vanish.

The connection to the standard formulation of quantum field theory is arrived at by evaluating the equal fifth-time expectation values. That is, it is possible to prove that

$$
\lim _{t \rightarrow \infty}\left\langle\phi\left(x_{1}, t\right) \phi\left(x_{2}, t\right) \ldots \phi\left(x_{n}, t\right)\right\rangle_{n}=\frac{\int D \phi \phi\left(x_{1}\right) \phi\left(x_{2}\right) \ldots \phi\left(x_{n}\right) e^{-s|\phi|}}{\int D \phi e^{-s(\phi)}}
$$


where $S[\phi]$ is the four dimensional action. Note that on the right hand side of the equation the field, $\phi$, is a function of the four physical space-time dimensions, while on the left hand side of the equation, $\phi$ is a function of the five dimensional extended space. By starting the Langevin system at $t_{0}=-\infty$, the system is equilibrated for any finite fifth-time, so there is no need to take the limit of infinite fifth-time to make the correspondence to the standard formulation of field theory.

There are quite a few proofs in the literature of the equivalence of stochastic quantization to the standard procedures of quantization. One way to make the connection is by defining the Fokker-Planck probability [36], which deacribes the probability density of finding the field $\phi$ at a given value under the Langevin dynamica. By deriving an evolution equation for the FokkerPlanck probability, it is possible to show that for essentially arbitrary initial conditions, at equilibrium, the Fokker-Plandk probability reduces to the probability density of the urdinary formulation. There are also proofs based on the various perturbative expansions of stochastic quantization [37]. Another rather elegant proof makes use of a hidden supersymmetry [38].

The Langevin equation ean be used to perturbatively solve quantum field theories. In general, the lagrangian will consist of a kinetic term plus an interaction potential. Thus, the Langevin equation is

$$
\frac{\partial \phi(x, t)}{\partial t}+\left(-\partial^{2}+m^{2}\right) \phi(x, t)=-V^{\prime}(\phi(x, t))+\eta(x, t)
$$


where $V^{\prime}(\phi)$ is the derivative of the potential with respect to the field $\phi$. One way to handle this equation is with the method of Green functions.

$$
\frac{\partial G\left(x-x^{\prime}, t-t^{\prime}\right)}{\partial t}+\left(-\partial^{2}+m^{2}\right) G\left(x-x^{\prime}, t-t^{\prime}\right)=\delta^{4}\left(x-x^{\prime}\right) \delta\left(t-t^{\prime}\right)
$$

The causal Green function in coordinate space is

$$
G\left(x-x^{\prime}, t-t^{\prime}\right)=\theta\left(t-t^{\prime}\right) \int \frac{d^{4} p}{(2 \pi)^{4}} e^{-i p\left(x-x^{\prime}\right)} e^{-\left(p^{2}+m^{2}\right)\left(t-t^{\prime}\right)}
$$

The Green function can be uned to rewrite the differential equation as an integral equation,

$$
\phi(x, t)=\int d^{t} x^{\prime} \int_{-\infty}^{\infty} d t^{\prime} G\left(x-x^{\prime}, t-t^{\prime}\right)\left[\eta\left(x^{\prime}, t^{\prime}\right)-V^{\prime}\left(\phi\left(x^{\prime}, t^{\prime}\right)\right)\right],
$$

that contains the initial condition that the field vanishes at $t_{0}=-\infty$, as well as the causality requirement. To simpiify matters, a compact notation is introduced.

$$
G_{21} \equiv G\left(x-x_{1}, t-t_{1}\right), \quad \eta_{1} \equiv \eta\left(x_{1}, t_{1}\right), \quad \int_{1} \equiv \int d^{4} x_{1} \int_{-\infty}^{\infty} d t_{1}
$$

By ileration the integral equation (1.8) can be solved as a perturbative series.

$$
\phi(x, t)=\int_{1} G_{21} \eta_{1}-\int_{1} G_{21} V^{\prime}\left(\int_{2} G_{12} \eta_{2}-\int_{2} G_{12} V^{\prime}\left(\int_{9} G_{23} \eta_{5}-\cdots\right)\right) .
$$

An explicit example of how the the Langevin equation can be used to generate a perturbation series is the massive scalar $\phi^{4}$ theory. To the first 
order in the coupling constant the field is given from equation (1.10) to be

$$
\phi(x, t)=\int_{1} G_{21} \eta_{1}-\frac{\lambda}{3 !} \int_{1} G_{21}\left[\int_{2} G_{12} \eta_{2}\right]^{3}+\cdots
$$

The tree diagrams corresponding to the perturbation series are given in Fig. II-1. Each line corresponds to a Green function, while the crosses at the ends of the diagrams represent the noise term, $\eta$. The vertex factors are the same an for ordinary Feynman diagrams, up to a possible combinatoric factor.

The loop diagrams come about by piecing together the tree diagrams (Fig. II-2). For example, the two point correlation function is

$$
\begin{aligned}
\left\langle\phi(x, t) \phi\left(x^{\prime}, t^{\prime}\right)\right\rangle_{\eta}= & \left\langle\int_{1} \int_{2} G_{z 1} G_{\left.x^{\prime} \eta_{1} \eta_{1} \eta_{2}\right\rangle_{\eta}}\right. \\
& -\frac{\lambda}{3 !}\left\langle\int_{1} \int_{2}\left[G_{21} G_{z^{\prime} 2}+G_{x^{\prime} 1} G_{22}\right] \eta_{1}\left[\int_{3} G_{29} \eta_{3}\right]^{3}\right\rangle_{\eta}+O\left(\lambda^{3}\right) .
\end{aligned}
$$

From equation (1.2), the n-point $\eta$ correlation functions are sums of products of delta-functions. The delta-functions can be thought of as glue that holds the tree diagrams together to form the n-point $\phi$ correlations. As will be discussed in the next section, stochastic regularization consists of smearing the delta-function glue in fifth-time.

The zeroth order contribution is given by

$$
\left\langle\phi(x, t) \phi\left(x, t^{\prime}\right)\right\rangle_{\eta}^{(0)} \equiv D\left(x-x^{\prime}, t-t^{\prime}\right)
$$




$$
\begin{aligned}
& =2 \int_{1} G_{21} G_{z^{\prime} 1} \\
& =\int \frac{d^{4} p}{(2 \pi)^{4}} e^{-i p\left(z-z^{\prime}\right)} \frac{e^{-\left(p^{2}+m^{2}\right)\left|t-t^{\prime}\right|}}{p^{2}+m^{3}} .
\end{aligned}
$$

Therefore, in momentum space, the zeroth order free propagator is given by

$$
D_{12}(p)=\frac{e^{-\left(p^{2}+m^{2}\right)\left|t_{1}-t_{2}\right|}}{p^{2}+m^{2}}
$$

where the subscript on $D_{12}(p)$ refers only to the fifth-time coordinate. After replacing the $\eta$ correlations with the appropriate delta-functions and combining terms that differ only by dummy indices, the first order contribution is given by

$$
\left\langle\phi(x, t) \phi\left(x^{\prime}, t^{\prime}\right)\right\rangle_{\eta}^{(1)}=-2 \lambda \int_{1} \int_{3} \int_{3}\left[G_{21} G_{z^{\prime} 3} G_{21} G_{29} G_{23}+G_{z^{\prime} 1} G_{22} G_{21} G_{23} G_{29}\right] .
$$

By explicit evaluation, it is easy to check that for $t=t$, the same result is obtained as by using ordinary Feynman diagrams. 


\section{Chapter III: Stochastic Regularization of Scalar}

\section{Electrodynamics}

This chapter discusses the stochastic regularization $[17,18]$ of scalar electrodynamics, further details of which can be found in Ref. [19]. The infinite part of the photon self energy is calculated to one loop order using the stochastic regularizer and the infinite part of the photon vacuum polarization tensor is shown automatically to come out transverse, as it should. The photon does not acquire a mase at the one-loop level, because at zero external momentum the photon vacuum polarization is shown to vanish. By a diagrammatic analysis, it is shown that the Ward identity that equates the scalar wavefunction renormalization, $Z_{2}$ to the one photon vertex renormalization, $Z_{1}$, holds to all orders of the stochastically regularized theory. 


\section{Stochastic Regulariration}

Since an extra dimension is present in the Langevin approach, the infinities can be smeared without destroying any relevant symmetries of the corresponding four dimensional theory. The preservation of the symmetries that are present in the infinite theory is crucial to finding a satisfactory regularization scheme. A time smeared system is known as a non-Markovian system [9]. In general, such a system can be expeeted to be less divergent than its Markovian counterpart. From the perturbative point of view, stochastic regularization can be thought of as preventing the loops of the correlation functions from completely closirg on themselves in the fifth-time.

There are at least two choices for fifth-time smearing the Langevin sys. tem. Either the Langevin equation or the probability distribution of the random aoies, $\eta$, can be smeared. By otudying the first order correction in the $\lambda \phi^{4}$ theory, it is poasible to show that the non-Markovian Langevin equation,

$$
\frac{\partial \phi(x, t)}{\partial t}=-\int d t^{\prime} \alpha_{A}\left(t-t^{\prime}\right) \frac{\delta S[\phi]}{\delta \phi\left(x, t^{\prime}\right)}+\eta(x, t)
$$

where $\alpha_{4}$ in a smearing function, can at best only remove two degrees of divergence in the perturbation theory. Quadratically divergent integrals become logarithmically divergent, and there does not exist a regularization function that does better.

The other possibility is to smear the $\eta$ probability functional $[17,18]$. 
In this scheme, the Langevin equation is left alone, while equation (1.2) of chapter II is replaced by

$$
\langle F[\phi(\eta)\}\rangle_{\eta} \equiv \frac{\int D_{\eta} F[\phi(\eta)] \exp \left(-\frac{1}{4} \int \eta(x, t) \alpha_{A}^{-1}\left(t-t^{\prime}\right) \eta\left(x, t^{\prime}\right) d^{4} x d t d t^{\prime}\right)}{\int D_{\eta} \exp \left(-\frac{1}{4} \int \eta(x, t) \alpha_{A}^{-1}\left(t-t^{\prime}\right) \eta\left(x, t^{\prime}\right) d^{4} x d t d t^{\prime}\right)} .
$$

This changes the $\eta$ correlation to

$$
\left\langle\eta(x, t) \eta\left(x^{\prime}, t^{\prime}\right)\right\rangle_{\eta}=2 \delta^{4}\left(x-x^{\prime}\right) \alpha_{A}\left(t-t^{\prime}\right)
$$

The smeariug functions $\alpha_{A}$ and $\alpha_{A}^{-1}$ are functional inverses of each other, in the wave that

$$
\int d t^{\prime \prime} \alpha_{\Delta}\left(t-t^{\prime \prime}\right) \alpha_{\Delta}^{-1}\left(t^{\prime \prime}-t^{\prime}\right)=\delta\left(t-t^{\prime}\right)
$$

The hope is that, because

$$
\lim _{\Delta \rightarrow \infty} \alpha_{A}\left(t-t^{\prime}\right)=\delta\left(t-t^{\prime}\right)
$$

as $\Delta$ becomes intinite, the original theory is recovered with all relevant symmetries intact.

Since the Langevin equation is unaffected by the stochastic regularization, the physical feld is the same as in the unregularized case, so that

$$
\left\langle\phi(x, t) \phi\left(x^{\prime}, t^{\prime}\right)\right\rangle_{\eta}^{(0)}=\int_{2} \int_{2} G_{21} G_{x^{\prime 2}}\left\langle\eta_{1} \eta_{2}\right\rangle_{\eta}
$$

In this case, however, the two point $\eta$ correlation is given by equation (1.3). 
Working in physical momentum space the zeroth order propagator is

$$
\begin{aligned}
& D_{12}^{A}(p) \equiv 2 \int d t_{3} \int d t_{4} G_{19}(p) G_{24}(p) \alpha_{A}\left(t_{3}-t_{4}\right) \\
& =2 \int_{-\infty}^{t_{1}} d t_{3} \int_{-\infty}^{t_{2}} d t_{1} e^{-\left(t_{1}-t_{1}\right)\left(p^{2}+m^{2}\right)} e^{-\left(t_{2}-t_{4}\right)\left(p^{2}-m^{2}\right)} \alpha_{A}\left(t_{3}-t_{4}\right) \\
& =2 \int \frac{d E}{2 \pi} e^{-i E\left(t_{1}-t_{2}\right)} \frac{\alpha_{A}(E)}{\left(p^{2}+m^{2}\right)^{2}+E^{2}},
\end{aligned}
$$

where the Fourier transform of the smearing function, $\alpha_{A}(E)$, has been introduced. Since there is an extra power of $p^{2}$ in the denominator over the ordinary Feynman propagator, a reduction of two degrees of divergence can be obtained, if $\alpha_{A}(E)$ cuts off for large values of $E$. Since all loops in the perturbative expansion of an arbitrary theory contain at least one factor of $\alpha_{A}(E)$, the logarithmically divergent loops can be expected to be rendered finite.

It is a little more difficult to regularize a theory whose diagrams are quadratically divergent. For example, the first order correction to the scalar propagator in $\phi^{4}$ theory, is

$$
\begin{aligned}
& \left\langle\phi\left(x_{1}, t_{2}\right) \phi\left(x_{2}, t_{2}\right)\right\rangle_{7}^{(1)}=-\frac{\lambda}{3 !}\left\langle\int _ { 3 } \int _ { 4 } \left[ G_{14} G_{25}+G_{19} G_{24}\left|\eta_{5}\left[\int_{5} G_{48} \eta_{8}\right]^{5}\right\rangle_{\eta}\right.\right. \\
& =-\frac{\lambda}{2} \int \frac{d^{4} k}{(2 \pi)^{4}} e^{-i k\left(x_{1}-z_{3}\right)} \int d t_{3}\left[D_{1 s}^{A}(k) G_{2 s}(k)\right. \\
& \left.+D_{2 s}^{A}(k) G_{1 s}(k)\right] \int \frac{d^{4} p}{(2 \pi)^{4}} D_{s s}^{A}(p) .
\end{aligned}
$$

It is possible to find a necessary condition on the set of functions that can 
be used as regularizers by studying the loop of the first order correction [17]. In this case, the loop is decoupled from the rest of the diagram, so the loop can be studied by itself. The loop is given by

$$
\begin{aligned}
L & =\int \frac{d^{4} p}{(2 \pi)^{4}} D_{33}^{A}(p) \\
& =2 \int_{0}^{\infty} d t_{4} \int_{0}^{\infty} d t_{6} \int \frac{d^{4} p}{(2 \pi)^{4}} e^{-\left(1_{4}+t_{4}\right)\left(p^{2}+m^{2}\right)} \alpha_{A}\left(t_{4}-t_{8}\right) \\
& \sim \frac{2 \alpha_{A}(0)}{(4 \pi)^{2}} \int_{0}^{4} d T \frac{1}{T} .
\end{aligned}
$$

In order for the integral to be finite, a necessary condition on the regularization function is that [1T]

$$
\alpha_{A}(0)=0
$$

Uaing the Fourier transform of the smearing function, $\alpha_{\Delta}(E)$, condition (1.12) can be rewritten as

$$
\int \frac{d E}{2 \pi} \alpha_{A}(E)=0
$$

Therefore, to remove quadratic divergences, the support of $\alpha_{A}(E)$ is not positive. The generating functional in Euclidean space, in general, won't be well defined as can be seen by looking at the generating functional written in terms of the Fourier transformed fields.

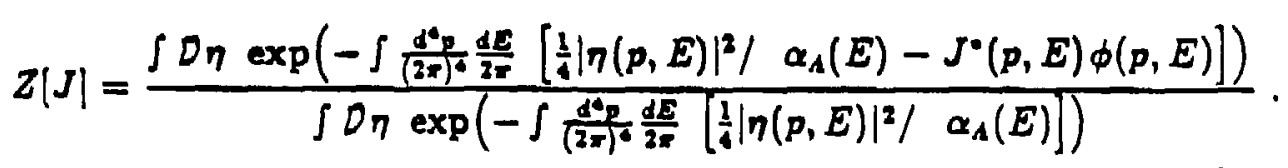


This action is unbounded from below, which seems to rule out the nonperturbative usefulneas of the stochastic regularizer for quadratically divergent theories [39]. For logarithmically divergent theories, such as supersymmetric theories, the nonperturbative usefulness of the stochastic regularizer is not ruled out. 
2. Stochastic Regularization of Perturbative Scalar Electrodynamics

The manifestly covariant gauge-fixed four dimensional action of euclidean scalar electrodynamics is

$S\left[A_{0}, \phi^{*}, \phi\right]=\int d^{+} x\left[-\frac{1}{2} A_{\mu}\left(T_{\mu \nu}+\frac{1}{\alpha} L_{\mu \nu}\right) \partial^{2} A_{\nu}+\left|\left(\partial_{\mu}-i e A_{\mu}\right) \phi\right|^{2}+m^{2}|\phi|^{2}\right]$.

Using the standard Feynman diagrammatical techniques, the quantum corrections to the vacuum polarization in scalar electrodynamics can easily be calculated. In doing the calculation, care must be taken, because the diagrams are infinite. For example, the first order correction to the vacuum polarization in euclidean space is given by (Fig. III-1):

$$
\Pi_{\mu \nu}(k)=-2 e^{2} \delta_{\mu \nu} \int \frac{d^{4} p}{(2 \pi)^{4}} \frac{1}{p^{2}+m^{2}}+e^{2} \int \frac{d^{4} p}{(2 \pi)^{4}} \frac{(2 p+k)_{\mu}(2 p+k)_{\nu}}{\left[(k+p)^{2}+m^{2}\right]\left(p^{2}+m^{2}\right)} .
$$

Using a naive momentum cutof, $\Delta$, on the integrals, to leading order in the cutoff, one obtains

$$
\Pi_{\mu \nu}(k) \sim-\frac{e^{2} \Delta^{2}}{16 \pi^{2}} \delta_{\mu}
$$

Thus, this naive regularizer explicitly breaks gauge invariance by giving the photon a mass.

An example of a well known gauge invariant regularization scheme is dimensional regularization [40]. In this scheme the dimension of space-time is "analytically continued" to $4-\epsilon$ dimensions, where the integral is finite. In this case, the photon mass contribution of each diagram just vanish to 
give a gauge invasiant vacuum polarization,

$$
\Pi_{\nu}(k)=\frac{1}{3} \frac{e^{2}}{(4 \pi)^{2}}\left(k_{\mu} k_{\nu}-k^{2} \delta_{\mu \nu}\right) \ln \frac{A^{2}}{m^{2}}+\text { regular terms }
$$

where the usual connection, $\frac{2}{a} \rightarrow \ln \Lambda^{2}$, has been made and where $\Lambda$ is a cutoff parameter with units of momentum.

As first discussed by Parisi and Wu [8], it is possible to formulate gauge theories without the need for gauge-fixing, by using atochastic quantization. The gauge invariance manifests itself by a nonequilibrating random walk in the gauge parameter space. Since the physically interesting quantities are gauge invariant, the wandering in the gauge parameter space is essentially irrelevant. In fact, as Parisi and Wu pointed out, it is possible to rewrite the Langevin equations in terms of gauge invariant fields. Another simple way to avoid the nonequilibration of the abelian gauge field is by intzoducing a simple gauge-fixing term, sinct the property that gauge-fixing is unnecessary is unimportant for this study of regularization.

The Langevin equations of the gauge-fixed scalar electrodynamics are

$$
\begin{gathered}
\frac{\partial \phi}{\partial t}=\left(\partial^{2}-m^{2}\right) \phi-i e A_{\mu} \partial_{\mu} \phi-i e \partial_{\mu}\left(A_{\mu} \phi\right)-e^{2} A_{\mu} A_{\mu} \phi+\eta \\
\frac{\partial \phi^{*}}{\partial t}=\left(\partial^{2}-m^{2}\right) \phi^{*}+i e A_{\mu} \partial_{\mu} \phi^{\bullet}+i e \partial_{\mu}\left(A_{\mu} \phi^{*}\right)-e^{2} A_{\mu} A_{\mu} \phi^{\bullet}+\eta^{*} \\
\frac{\partial A_{\mu}}{\partial t}=\left(T_{\mu \nu} \partial^{2}+\frac{1}{\alpha} L_{\mu \nu} \partial^{2}\right) A_{\nu}-i e \phi^{\bullet}\left(\vec{\partial}_{\mu}-\bar{\partial}_{\mu}\right) \phi-2 e^{2} A_{\mu} \phi^{\bullet} \phi+\eta_{\mu},
\end{gathered}
$$


with unsmeared expectation values defined by

$$
\left\langle F\left[A_{\sigma}, \phi^{*}, \phi\right]\right\rangle_{\eta} \equiv \frac{\int D \eta_{\mu} D \eta^{*} D \eta F\left[A_{\sigma}, \phi^{*}, \phi\right] \exp \left(-\frac{1}{4} \int\left[\eta_{\nu}^{2}+2 \eta^{*} \eta\right] d^{4} x d t\right)}{\int D \eta_{\mu} D \eta^{*} D \eta \exp \left(-\frac{1}{4} \int\left[\eta_{\nu}^{2}+2 \eta^{*} \eta\right] d^{4} x d t\right)} .
$$

The causal Green function for the photon Langevin equation is

$$
G_{\mu \nu}(x, t)=\theta(t) \int \frac{d^{4} k}{(2 \pi)^{4}} e^{-i k x}\left[T_{\mu \nu}(k) e^{-k^{2} t}+L_{\mu \nu}(k) e^{-k^{2} t / \alpha}\right]
$$

while in the unregularized theory the zeroth order propagator is

$$
\begin{aligned}
D_{\mu \nu}(x, t) & \equiv\left\langle A_{\mu}(x, t) A_{\nu}(0,0)\right\rangle_{\eta}^{(0)} \\
& =\int \frac{d^{4} k}{(2 \pi)^{4}} e^{-i k x}\left[\frac{T_{\mu \nu}(k)}{k^{2}} e^{-k^{2} t}+\frac{\alpha L_{\mu \nu}(k)}{k^{2}} e^{-k^{2} t / \alpha}\right],
\end{aligned}
$$

where $T_{\mu \nu}(k)$ and $L_{\mu \nu}(k)$ are respectively the transverse and longitudinal projection operators. The two point functions for the scalars are given in equations (1.7) and (1.14) of chapter ПI. As with ordinary Feynman diagrammatic calculations the simplest gauge to we is Feynman gauge, where $\alpha=1$. Henceforth, the Feynman gauge will be used exclusively.

An example of a function that satisties the condition of equation (1.12), and renders the loops finite, is [17]

$$
\alpha_{A}^{(d)}\left(t-t^{\prime}\right)=\frac{A^{4}\left|t-t^{\prime}\right|}{2} e^{-A^{2}\left|t-t^{\prime}\right|}
$$

The superscript refers to the fact that the Fourier transform of the above regularization function has a double pole structure. For calculational purposes it is easier to use a function whose Fourier transform has a single pole 
structure. Namely,

$$
\alpha_{\Lambda}^{(0)}\left(t-t^{\prime}\right)=\frac{A^{2}}{2} e^{-A^{2}\left|t-t^{\prime}\right|}
$$

which does not satisfy the requirements of a quadratic divergence regularization function. The two functions are related by

$$
\alpha_{A}^{(d)}\left(t-t^{\prime}\right)=-A^{+} \frac{\partial}{\partial A^{2}}\left[\frac{\alpha_{A}^{(d)}\left(t-t^{\prime}\right)}{\Delta^{2}}\right] .
$$

Therefore, $a^{(0)}$ can be used until a divergent integral is to be evaluated, where equation (2.11) will be used to replace $\alpha_{A}^{(0)}$ with $\alpha_{\Lambda}^{(d)}$, within the calculation.

Since this section is only concerned with the perturbative one loop expansion of the photon propagator, the photon random noise field need not be fifth-time smeared, since only scalars appear within the loops. Using equations (1.8) and (2.10), the zeroth order regularized scalar two point function is

$$
\begin{gathered}
D_{12}^{(e)}(p)=e^{-\left|t_{1}-t_{2}\right|\left(p^{2}+m^{2}\right)}\left[\frac{\Delta^{2}}{\left(p^{2}+m^{2}\right)\left(p^{2}+m^{2}+\Delta^{2}\right)}-\frac{\Delta^{2}}{\left(p^{2}+m^{2}\right)^{2}-\Delta^{4}}\right] \\
+e^{-\Delta^{2}\left|s_{1}-t_{2}\right|} \frac{\Lambda^{2}}{\left(p^{2}+m^{2}\right)^{2}-\Delta^{4}} .
\end{gathered}
$$

Note that the apparent singularity at $p^{2}+m^{2}= \pm d^{2}$ is fictitious.

The seven Langevin diagrams of the one-loop correction to the photon propagator in scalar electrodynamics are given in Fig. III-2. Since only physical expectation values are of interest, the external fifth-times are taken 
to be equai. Introdueing the simplifying notation

$$
a \equiv p^{2}+m^{2}, \quad b \equiv(p+k)^{2}+m^{2},
$$

the diagrams with no external momenta in the loop (Fig. III-2a) are given by

$$
\begin{aligned}
P_{\alpha^{(d)}}^{1}(k)= & -4 e^{3} \delta_{\mu \nu} \int d t_{1} D_{01}^{\infty}(k) G_{01}^{o x}(k) \\
& \quad \times \int \frac{d^{4} p}{(2 \pi)^{4}}\left(-\Delta^{4} \frac{\partial}{\partial \Delta^{2}}\right)\left\{\frac{D_{11}^{(o)}(p)}{\Delta^{2}}\right\} \\
= & -\frac{2 e^{2} \delta_{\omega}}{k^{4}} \int \frac{d^{4} p}{(2 \pi)^{4}} \frac{\Delta^{4}}{a\left(a+\Delta^{2}\right)^{2}} .
\end{aligned}
$$

The other diagrams are significantly more complicated because of the intertwining of the external legs with the loop. In order to simplify the expressions, the vertex factors will be written as

$$
V_{\mu \nu} \equiv e^{2}(2 p+k)_{\mu}(2 p+k)_{\nu}
$$

The diagram in Fig. III-2b is given by

$$
\begin{aligned}
& P_{\infty}^{(d)^{2}}(k)=\int d t_{1} \int d t_{2} G_{01}^{\infty}(k) G_{02}^{\infty y}(k) \int \frac{d^{4} p}{(2 \pi)^{4}} V_{\mu \nu} D_{12}^{(d)}(p+k) D_{12}^{(d)}(p) \\
& =\frac{\delta_{\rho \mu} \delta_{o v}}{k^{2}}\left(-\Lambda_{1}^{4} \frac{\partial}{\partial \Lambda_{1}^{2}}\right)\left(-\Lambda_{2}^{4} \frac{\partial}{\partial \Lambda_{2}^{2}}\right) \int \frac{d^{4} p}{(2 \pi)^{4}} V_{\mu \nu} \frac{1}{b^{2}-\Lambda_{1}^{4}} \frac{1}{a^{2}-\Lambda_{2}{ }^{4}} \\
& \times\left[\frac{\Lambda_{1}^{2} \Delta_{2}^{2}}{a b\left(a+b+k^{2}\right)}-\frac{\Lambda_{2}^{2}}{a\left(a+k^{2}+\Lambda_{1}^{2}\right)}-\frac{\Lambda_{1}^{2}}{b\left(b+k^{2}+\Lambda_{2}^{2}\right)}\right. \\
& \left.+\frac{1}{\left(k^{2}+\Lambda_{1}^{2}+\Lambda_{2}^{2}\right)}\right]\left.\right|_{A_{1}=A_{2}=A} \\
& =\frac{\delta_{\mu \nu} \delta_{\sigma \nu}}{k^{4}}\left(-\Lambda_{1}^{4} \frac{\partial}{\partial \Lambda_{1}^{2}}\right)\left(-\Lambda_{2}^{4} \frac{\partial}{\partial \Lambda_{2}^{2}}\right) \int \frac{d^{4} p}{(2 \pi)^{4}} V_{\mu \nu} k^{2}
\end{aligned}
$$




$$
\begin{aligned}
& \times\left[\left(\Delta_{1}^{2}+a+b+k^{2}\right) \Delta_{2}^{4}+\left(\Delta_{2}^{2}+a+b+k^{2}\right) \Delta_{1}^{4}\right. \\
& +\left(3 k^{2}+2 b+2 a\right) \Delta_{1}^{2} \Delta_{2}^{2}+k^{2}\left(2 k^{2}+3 b+3 a\right)\left(A_{1}^{2}+A_{2}^{2}\right) \\
& +(a+b)^{2}\left(\Delta_{1}^{2}+\Delta_{2}^{2}\right)+k^{4}\left(k^{2}+2 b+2 a\right) \\
& \left.+k^{2}\left(a^{2}+3 a b+b^{2}\right)+a b^{2}+a^{2} b\right] \\
& /\left[a b\left(a+b+k^{2}\right)\left(\Delta_{1}^{2}+b\right)\left(\Delta_{2}^{2}+a\right)\left(\Delta_{1}^{2}+a+k^{2}\right)\right. \\
& \left.\quad \times\left(\Delta_{2}^{2}+b+k^{2}\right)\left(\Delta_{1}^{2}+\Delta_{2}^{2}+k^{2}\right)\right]\left.\right|_{A_{1}=A_{2}=A},(2.16 \mathrm{c})
\end{aligned}
$$

where the two regularization parameters are distinguished, in order to be able to differentiate individually each of the two regularization functions contained within the diagram. Later $A_{1}$ will be set equal to $A_{2}$. The diagrams in Fig. III-2e contribute a value of

$$
\begin{aligned}
& P_{\infty}^{(\infty)}(k)=2 \int d t_{1} \int d t_{2} D_{01}^{\infty}(k) G_{02}^{\infty v}(k) \\
& \times \int \frac{d^{4} p}{(2 \pi)^{4}} V_{\mu \nu} D_{12}^{(d)}(p+k) G_{21}(p) \\
& =\frac{\delta_{o \mu} \delta_{\alpha u}}{k^{4}} \int \frac{d^{4} p}{(2 \pi)^{4}} V_{\mu \nu}\left(-\Delta^{4} \frac{\partial}{\partial A^{2}}\right)\left\{\frac{1}{b^{2}-\Delta^{4}}\right. \\
& \left.\times\left[\frac{1}{k^{2}+a+\Delta^{2}}-\frac{A^{2}}{b\left(k^{2}+a+b\right)}\right]\right\} \\
& =\frac{\delta_{\omega \omega_{\sigma \nu}} \delta_{\sigma}}{k^{4}} \int \frac{d^{4} p}{(2 \pi)^{4}} V_{\mu \nu}\left(-\Lambda^{4} \frac{\partial}{\partial \Lambda^{2}}\right) \\
& \times \frac{\left(k^{2}+a+b+A^{2}\right)}{b\left(b+A^{2}\right)\left(a+b+k^{2}\right)\left(a+k^{2}+A^{2}\right)} .
\end{aligned}
$$

Similarly the last two diagrams can be evaluated. The values are identical 
to the diagrams just caleulated, as can be shown either by symmetry or by shifting the variables of integration. Therefore, the diagrams in Fig. III-2d contribute a value of

$$
P_{p o}^{(d)^{4}}(k)=P_{p 0^{(d)}}^{(k)} \text {. }
$$

In order to make the theory finite the results obtained by using $\alpha_{A}^{(0)}$ are taken and differentiated in order to obtain the results by using $\alpha_{A}^{(d)}$. For calculational purposes it is better to use the form of the vacuum polarization that contains no apparent singularities. After truncating the external photon lines the vacuum polarization of the photon is

$$
\Pi_{\mu \nu}^{(d)}(k) \equiv \Pi_{\mu \nu}^{(d)}{ }^{1}(k)+\Pi_{\mu \nu}^{(d)^{2}}(k)+\Pi_{\mu \nu}^{(d)^{3}}(k)+\Pi_{\mu \nu}^{(d)^{4}}(k)
$$

where

$$
\begin{aligned}
& \Pi_{\omega}^{(d)}(k)=-2 e^{2} \delta_{\mu \nu} \int \frac{d^{4} p}{(2 \pi)^{4}} \frac{\Delta^{4}}{a\left(a+\Delta^{2}\right)^{2}}, \\
& \Pi_{\mu \nu}^{(d)^{2}}(k)=k^{2}\left(-\Delta_{1}{ }^{4} \frac{\partial}{\partial \Delta_{1}^{2}}\right)\left(-\Delta_{2}{ }^{4} \frac{\partial}{\partial \Delta_{2}^{2}}\right) \int \frac{d^{4} p}{(2 \pi)^{4}} V_{\mu \nu} \\
& \times\left[\left(\Lambda_{1}^{2}+a+b+k^{2}\right) \Delta_{2}^{4}+\left(\Lambda_{2}^{2}+a+b+k^{2}\right) \Lambda_{1}^{4}\right. \\
& +\left(3 k^{2}+2 b+2 a\right) \Delta_{1}^{2} \Delta_{2}^{2}+k^{2}\left(2 k^{2}+3 b+3 a\right)\left(A_{1}^{2}+A_{2}^{2}\right) \\
& +(a+b)^{2}\left(A_{1}^{2}+\Delta_{2}{ }^{2}\right)+k^{4}\left(k^{2}+2 b+2 a\right) \\
& \left.+k^{2}\left(a^{2}+3 a b+b^{2}\right)+a b^{2}+a^{2} b\right] \\
& /\left[a b\left(a+b+k^{2}\right)\left(\Lambda_{1}^{2}+b\right)\left(\Lambda_{2}^{2}+a\right)\left(\Lambda_{1}^{2}+a+k^{2}\right)\right.
\end{aligned}
$$




$$
\begin{aligned}
& \left.\times\left(\Delta_{2}^{2}+b+k^{2}\right)\left(\Delta_{1}^{2}+\Delta_{2}^{2}+k^{2}\right)\right]\left.\right|_{A_{1}=A_{2}=A} \\
& \Pi_{\mu \nu}^{(d)^{3}}(k)=e^{3} \int \frac{d^{4} p}{(2 \pi)^{4}} \frac{\Lambda^{4}(2 p+k)_{\mu}(2 p+k)_{\nu}}{b\left(b+A^{2}\right)\left(a+b+k^{2}\right)\left(a+k^{2}+A^{2}\right)} \\
& \times\left[\frac{a+b+k^{2}+\Delta^{2}}{b+A^{2}}+\frac{a+b+k^{2}+A^{2}}{a+k^{2}+\Lambda^{2}}-1\right] \text {, } \\
& \Pi_{\mu \nu}^{(d)}(k)=e^{2} \int \frac{d^{4} p}{(2 \pi)^{4}} \frac{A^{4}(2 p+k)_{\mu}(2 p+k)_{\nu}}{a\left(a+\Delta^{2}\right)\left(a+b+k^{2}\right)\left(b+k^{2}+A^{2}\right)} \\
& \times\left[\frac{a+b+k^{2}+A^{2}}{a+\Delta^{2}}+\frac{a+b+k^{2}+\Delta^{2}}{b+k^{2}+\Delta^{2}}-1\right] \text {. }
\end{aligned}
$$

Although these integraly may seem quite formidable, only a few of the terms will contribute to the infinite part of the vacuum polarization.

A fundamental consequence of the gauge invariance of scalar electrodynamics is that the photon does not acquire a mass by the higher order corrections to the vacuum yolarization. Setting the external momentum to zero, the exact mass correction to the photon can be found. Explicitly,

$$
\Pi_{\mu \mu}^{(d)}(0)=\Pi_{\mu \mu}^{(d)^{2}}(0)+2 \Pi_{\mu \mu}^{(d)^{3}}(0)
$$

where,

$$
\Pi_{m}^{(d)}(0)=-8 e^{2} \int \frac{d^{4} p}{(2 \pi)^{4}} \frac{A^{4}}{\left(p^{2}+m^{2}\right)\left(p^{2}+m^{2}+A^{2}\right)^{2}}
$$

and

$$
\begin{aligned}
2 \Pi_{\mu \mu}^{(d)^{3}}(0)=4 A^{4} e^{2} \int \frac{d^{4} p}{(2 \pi)^{4}} \frac{p^{2}}{p^{2}+m^{2}} & {\left[\frac{1}{\left(p^{2}+m^{2}\right)\left(p^{2}+m^{2}+A^{2}\right)^{2}}\right.} \\
& \left.+\frac{2}{\left(p^{2}+m^{2}+A^{2}\right)^{3}}\right]
\end{aligned}
$$




$$
\begin{aligned}
& =4 \Lambda^{4} e^{2} \int_{0}^{1} d z \int_{0}^{\infty} \frac{d p^{2}}{(4 \pi)^{2}} \frac{6 z p^{4}}{\left(p^{2}+m^{2}+z \Lambda^{2}\right)^{4}} \\
& =8 \Lambda^{4} e^{2} \int_{0}^{1} d z \int_{0}^{\infty} \frac{d p^{2}}{(4 \pi)^{2}} \frac{2 z p^{2}}{\left(p^{2}+m^{2}+z \Lambda^{2}\right)^{3}} \\
& =8 e^{2} \int \frac{d^{4} p}{(2 \pi)^{4}} \frac{\Lambda^{4}}{\left(p^{2}+m^{2}\right)\left(p^{2}+m^{2}+\Lambda^{2}\right)^{2}} .
\end{aligned}
$$

Thus, the desired result is

$$
\Pi_{\mu \mu}^{(d)}(0)=0
$$

and the mass correction vanishes.

A direct evaluation of the finite parts of the vacuum polarization with the stochastic regularizer is fairly involved and will not be discussed here. We have computed only the infinite part of the vacuum polarization for nonzero external momentum. The contribution to the vacuum polarization of the simpleat diagrams is from equation (2.20).

$$
\begin{aligned}
\Pi_{m \nu}^{(d)}(k) & =-2 e^{2} \delta_{\mu \nu} \int \frac{d^{4} p}{(2 \pi)^{4}} \frac{\Delta^{4}}{\left(p^{2}+m^{2}\right)\left(p^{2}+m^{2}+\Delta^{2}\right)^{2}} \\
& =-\frac{e^{2}}{8 \pi^{2}} \delta_{\mu \nu}\left[\Delta^{2}-m^{2} \ln \left(\frac{m^{2}+\Delta^{2}}{m^{2}}\right)\right] .
\end{aligned}
$$

The next contribution is given by equation (2.21). By power counting, the integral in equation (2.21) is Gnite even before differentiating with respect to $d^{2}$. Note that the only possible singularity as $A \rightarrow \infty$ is logarithmic. In fact, since an ultraviolet divergence in $A^{2}$ can only occur when there is an infrared divergence in $m^{2}$, the terms with no such divergence in $m^{2}$ can immediately be eliminated as being finite. As a further simplification, $k^{2}$ can 
be set to zero within the integral, without affecting the leading order in $\Lambda^{2}$. Also $m^{2}$ can be be neglected except where it is needed to prevent an infrared divergence within the integral. After performing all these simplifications, equation (2.21) is reduced to

$$
\begin{aligned}
\Pi_{\mu \nu}^{(d)^{2}}(k)=\Lambda_{1}^{4} \Delta_{2}^{4} e^{2} k^{2} \delta_{\mu \nu} & \frac{\partial}{\partial \Lambda_{1}^{2}} \frac{\partial}{\partial \Delta_{2}^{2}} \int \frac{d^{4} p}{(2 \pi)^{4}} \frac{1}{2 p^{2}\left(p^{2}+m^{2}\right)} \\
& \times\left.\frac{\Lambda_{1}^{2} \Delta_{2}^{2}}{\left(p^{2}+\Delta_{1}^{2}\right)^{2}\left(p^{2}+\Delta_{2}^{2}\right)^{2}}\right|_{A_{1}=\Delta_{2}=4} \quad+\text { regular terms. }
\end{aligned}
$$

This integral can be evaluated with the usual Feynman parameterization to arrive at the result,

$$
\Pi_{\mu \nu}^{\left(\phi^{2}\right.}(k)=\frac{1}{2} \delta_{\mu \nu} \frac{e^{2} k^{2}}{(4 \pi)^{2}} \ln \frac{A^{2}}{m^{2}}+\text { regular terms, }
$$

where all terms that are finite as $A \rightarrow \infty$ have not been calculated. In the remaining contributions from equations (2.22) and (2.23), $k^{2}$ can be neglected compared to $\Delta^{2}$. As urual, this type of integral is done by first Feyman parameterization and then evaluating the momentum integrals. After neglecting all the terms that are finite as $A \rightarrow \infty$, the result is

$$
\begin{aligned}
\Pi_{\mu \nu}^{(d)^{3}}(k)= & \Pi_{\mu \mu}^{(d)}(k) \\
= & \frac{e^{2}}{(4 \pi)^{2}}\left[\delta_{\mu \nu}\left(\Delta^{2}-m^{2} \ln \frac{A^{2}}{m^{2}}-\frac{5}{12} k^{2} \ln \frac{A^{2}}{m^{2}}\right)+\frac{1}{6} k_{\mu} k_{\nu} \ln \frac{A^{2}}{m^{2}}\right] \\
& + \text { regular terms. }
\end{aligned}
$$

By adding everything together, the momentum independent pieces cancel 
and the infinite part of the one-loop vacuum polarization is found to be

$$
\Pi_{\mu \nu}^{(d)}=\frac{1}{3} \frac{e^{2}}{(4 \pi)^{2}}\left(k_{\mu} k_{\nu}-k^{2} \delta_{\mu \nu}\right) \ln \frac{A^{2}}{m^{2}}+\text { regular terms. }
$$

This is precisely the correct value, as was obtained by using dimensional regularization.

As discussed by Ishikawa [24], a modification in the identity

$$
\left\langle\phi\left(x_{1}\right) \frac{\delta S[\phi]}{\delta \phi\left(x_{2}\right)}\right\rangle=\delta^{4}\left(x_{1}-x_{2}\right)
$$

can oecur in stochantically regularized quadratically divergent acalar field theories. The leading behavior of quadratically divergent loops is proportional to $\Delta^{2}$, while the external legs of the Langevin diagrams may possess a $\Delta^{-2}$ dependence. The combination of these two factors can yield an extra Ginite nonzero contribution, in the limit that the cutoff becomes infinite.

Although it is not clear what the relevance of this fact is to regularization and renormalization, it is straightforward to show that no problem occurs at the one loop level in the gluon channel of stochastically regularized scalas electrodynamics. In order for there to be a possibility of modifying

$$
\left\langle A_{\mu}\left(x_{1}\right) \frac{\delta S\left[A_{\sigma}, \phi^{*}, \phi\right]}{\delta A_{\nu}\left(x_{2}\right)}\right\rangle=\delta_{\mu \nu} \delta^{4}\left(x_{1}-x_{2}\right)
$$

where $S\left|A_{0}, \phi^{*}, \phi\right|$ is the action of sealar electrodynamies $(2.1)$, the photon random noise feld should also be ffth-time smeared. Keeping only the leading behavior of the quadratically divergent loops, explicit calculation shows 
that there is no modification of identity (2.34). The coefficients of the various factors of $A^{2}$ that occur in the one-loop evaluation of the left hand side of equation (2.34) can be obtained by comparison to the results for the various contributions to the photon vacuum polarization. Just as the quadratic divergences proportional to $A^{2}$ have cancelled in the vacuum polarization, the quadratic divergences cancel in the explicit evaluation of the left hand side of (2.34), and no modification of the identity oceurs in the stochastic regularization of scalar electrodynamics. Of course, in the charged scalar channel, the identity analogous to (2.33) would again be quadratically divergent. In the case of pure Yang-Mills or QCD with fermions, one would expect the whole phenomenon to disappear, because all quadratic divergences are spurious. 


\section{Diagrammatic Proof of the Ward Identity to All Orders}

By working with the standard Feynman diagrams, it is possible to prove the Ward identity [41], (in Feynman gauge)

$$
\lim _{p \rightarrow 0} q^{2} V_{\sigma}(p, p-q)=-e \frac{\partial S(p)}{\partial p_{\sigma}}
$$

where $V_{\sigma}(p, p-q)$ is the complete three point function (Fig. III-3) and $S(p)$ is the complete scalar propagator (Fig. III-4). A regularization scheme that preserves this identity implies that $Z_{1}=Z_{2}$. The proof using the Langevin formulation is analogous to the proof using ordinary Feynman diagrams. The main difference is that there are two types of two point functions to consider. In the Langevin perturbative expansion, the external photon line can either be $D_{\mu \nu}^{12}(q)$ or $G_{\mu \nu}^{12}((q)$. In this section all orders of perturbation theory are being considered, so the probability density of the photon noise tield must also be fifth-time smeared. In Feynman gauge, the stochastically regularized two point functions, using $a_{\perp}^{(n)}$, are

$$
G_{\mu \nu}^{12}(q)=\delta_{\mu \nu} e^{-q^{2}\left(t_{1}-t_{3}\right)} \theta\left(t_{1}-t_{2}\right)
$$

and

$$
\begin{gathered}
D_{\omega \omega}^{12}(q)=-\delta_{\mu \nu} \Lambda^{4} \frac{\partial}{\partial \Lambda^{2}}\left[e^{-\left|t_{1}-t_{3}\right| q^{2}}\left(\frac{1}{q^{2}\left(q^{2}+\Lambda^{2}\right)}-\frac{1}{q^{4}-\Lambda^{4}}\right)\right. \\
\left.+e^{-A^{2}\left|\varepsilon_{1}-t_{2}\right|} \frac{1}{q^{4}-\Lambda^{4}}\right] .
\end{gathered}
$$

Since $G_{\mu \nu}^{12}(q)$ does not have a pole at $q^{2}=0$, vertex diagrams whose 
external photon line is $G_{\mu \nu}^{12}(q)$, do not contribute to the Ward identity (3.1). Vertex diagrams whose external photon line is $D_{\mu \nu}^{12}(q)$ do contribute, but in a simple way, because

$$
\lim _{q \rightarrow 0} q^{2} D_{\mu \nu}^{12}(q)=\delta_{\mu \nu}
$$

The proof of the Ward identity will proceed by showing that inserting a photon at zero momentum, $q$, at a given point in a typical Langevin diagram and then multiplying by $q^{2}$, is the same as differentiating that part of the Langevin diagram with respect to the momentum flowing through that point. Summing over all the possible ways to insert the photon into a diagram is therefore equal to summing over all the possible ways of differentiating with respect to the momentum flowing through the scalar lines. The sum over a elosed scalar loop vanishes, because the loop momentum is integrated over. Thus, only the derivatives with reapect to the momentum flowing through the sealar line that begins and ends externally are left. This is just the Ward identity (3.3).

All that remains to be done is to explicitly check that inserting a photon at zero momentum at a given point in a Langevin diagram is indeed equivalent to differentiating that part of the diagram with respect to the momentum flowing through the scalar. There are two types of scalar two point functions that appear within a typical Langevin diagram and one type 
of vertex factor that explicitly contain momentum dependence. Since

$$
\begin{gathered}
{\left[\frac{1}{1}\right] \equiv G_{12}(k)=e^{-\left(k^{2}+m^{2}\right)\left(t_{1}-t_{2}\right)} \theta\left(t_{1}-t_{2}\right),} \\
-e \frac{\partial}{\partial p_{0}} G_{12}(k)=2 e k_{0}\left(t_{1}-t_{3}\right) G_{13}(k),
\end{gathered}
$$

where $k=p-\sum p_{i}, p$ is the scalar line momentum, and the $p_{i}$ are the moments of internal photons that are attached to the scalar. On the other hand,

$$
\begin{aligned}
\lim _{i \rightarrow 0} q^{2}\left[\frac{k}{l_{q}^{2}}\right] & =2 e k_{a} \int d t_{3} G_{19}(k) G_{92}(k) \\
& =2 e k_{-}\left(t_{1}-t_{2}\right) G_{12}(k) .
\end{aligned}
$$

Therefore, diagrammatically,

$$
-e \frac{\partial}{\partial p_{0}}\left[\frac{1}{1}\right]=\lim _{i \rightarrow 0} q^{2}\left[\sqrt{1} \frac{h}{l_{q}^{2}}\right] .
$$

Similarly,

$$
D_{12}(k)=\int d t_{3} d t_{4} \alpha_{34}^{A} G_{1 s}(k) G_{24}(k)
$$

yields

$$
-e \frac{\partial}{\partial p_{0}}\left[\frac{1}{3_{4}}\right]=2 e k_{0} \int d t_{9} d t_{4} \alpha_{s i}^{A}\left[\left(t_{1}-t_{3}\right)+\left(t_{1}-t_{4}\right)\right] G_{19}(k) G_{34}(k) .
$$

Attaching the photon in the two possible ways results in

$$
\begin{aligned}
& \lim _{3 \rightarrow 0} q^{2}\left[\overrightarrow{d^{2}}+\frac{k}{\sqrt{f_{q}}}\right]=2 e k_{0} \int d t_{3} \int d t_{4} \int d t_{3} \alpha_{34}^{A} G_{13}(k) G_{33}(k) G_{34}(k) \\
& +2 e k_{a} \int d t_{9} \int d t_{4} \int d t_{B} \alpha_{34}^{A} G_{15}(k) G_{55}(k) G_{24}(k)
\end{aligned}
$$




$$
\begin{aligned}
=2 e k_{\sigma} \int d t_{3} \int d t_{1} & \alpha_{34}^{A} G_{1 s}(k) G_{2 s}(k) \\
& \times\left[\left(t_{2}-t_{1}\right)+\left(t_{1}-t_{3}\right)\right] .
\end{aligned}
$$

Thu, diagrammatically,

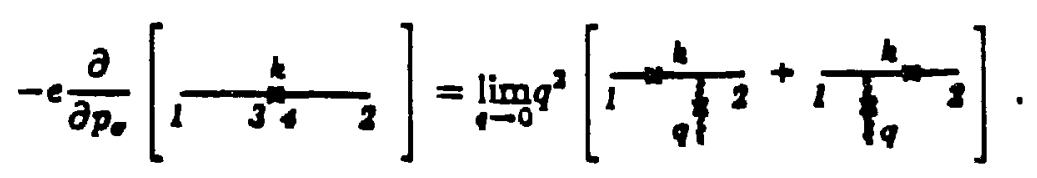

The reader may have noted that the fact that adding an external truncated photon to $D_{12}(k)$ is equivalent to differentiating with respect to the momentum fowing through the scalar is already contained in the fact that attaching an external truncated photon to $G_{12}(k)$ is equivalent to differentiating $G_{12}(k)$. However, the point was to explicitly show that the rsgularizer does not affect the results. Differentiating the one photon vertex factor yields the two photon vertex factor.

$$
-e \frac{\partial}{\partial p_{\sigma}}\left[e\left(2 p-2 \sum p_{i}-p_{j}\right)_{\mu}\right]=-2 e^{2} \delta_{\mu},
$$

or diagramanatically,

$$
-e \frac{\partial}{\partial p_{0}}\left[\bar{k}\left[p_{p_{1}^{k-p_{1}}}^{k}\right]=\lim _{q \rightarrow 0} q^{2}\left[\overline{p / p_{q}^{k-p_{1}}}\right],\right.
$$

where only the vertex factor is to be differentiated on the left hand side. Thus, it follows that the Ward identity (3.1) holds to all orders of perturbation theory. 


\section{Conclusions and Comments}

In this chapter we showed that the stochastic regularizer does, in fact, yield the correct gauge invariant infinite part of the one-loop photon vacuum polarization. The Ward identity that equates the scalar wavefunction renormalization to the one photon vertex renormalization was shown to hold to all orders of perturbation theory. Of course, it is possible that above the one-loop level, stochantic regularization breaks down, but the Ward identity would still hold. These results seem to indicate that the stochastic regularizer may be useful an a regularizer that preserves the symmetries and relevant identities that are present in the corresponding infinite theory.

Although no apparent difficulties appear in SED at the one-loop level, as we shall see in the next chapter, a serious problem arises when the stochastic regularization scheme in used in conjunction with Zwanziger's gauge-fixing. In terms of the attempt to construct a sonperturbative regularization, this incompatibility is quite serious since Zwanziger's gauge-fixing is the only known nonperturbative covariant gauge-fixing for nonabelian gauge theories. 


\section{Chapter IV: Incompatibility of Stochastic}

\section{Regularization and Zwanziger's Gauge Fixing}

\section{Stochastic Regularization with Zwanziger's Gauge Fixing}

Two outstanding developments in stochastic quantization [8] have been Zwanziger's gauge fixing $(10)$ and stochastic regularization $[17,18]$. The puspose of this chapter is to point out the failure of the synthesis of these two ideas, which leads to explicit gauge dependence in gauge invariant quantities. Furtber details can be found in Ref. [26].

In Yang-Mills theory, the two ideas are combined in the Langevin system

$$
\begin{gathered}
\dot{A}_{\mu}^{\eta_{\mu}}(x, t)=D_{\nu}^{a b} F_{\nu \mu}(x, t)+D_{\mu}^{a b} Z^{b}(x, t)+\eta_{\mu}^{a}(x, t) \\
\left\langle\eta_{\mu}^{a}(x, t) \eta_{\nu}^{b}\left(x^{\prime}, t^{\prime}\right)\right\rangle=2 \alpha_{\Lambda}\left(t-t^{\prime}\right) \delta^{\alpha \Delta} \delta_{\mu \nu} \delta^{4}\left(x-x^{\prime}\right),
\end{gathered}
$$

where $D_{\mu}^{\alpha}=\delta^{\alpha} \partial_{\mu}+g f^{\text {abe }} A_{\mu}^{e}$ is the covariant derivative. The $Z_{\text {wanziger }}$ function, $Z$, provides the gauge 6xing, while the fifth-time smearing $\alpha_{A}(t)$

$$
\int d t \alpha_{A}(t)=1, \quad \lim _{A \rightarrow \infty} \alpha_{A}(t)=\delta(t)
$$

provides the regularization. In the absence of gauge fixing, the stochastic regularization is manifestly gauge invariant, and presumably provides a perturbative ${ }^{2}$ regularization of gauge invariant quantities, since at least one

\footnotetext{
${ }^{3}$ The condition $a_{4}(0)=0$ is required for regularisation of theories with quadratic divergences, w diecussed in Chapter III. This is not a satiofactory non-perturbative regular-
} 
noise-regulated propagator appears in every closed loop. The Zwanziger term stops the random walk in gauge space, allowing the computation of gauge non-invariant quantities such as the Green functions. It is crucial, however, to demonstrate that gauge invariant observables have no $Z$-dependence.

A standard [42] Langevin argument seems to augur well for the synthesis. If $G^{\circ}$ is the generator of gauge transformations and $\Phi$ is a gauge invariant observable, then

$$
\begin{gathered}
\int d^{4} x Z^{a}(x, t) G^{a}(x, t) \Phi[A(\cdot, t)]=0, \\
G^{\circ}(x, t)=D_{\mu}^{\alpha} \frac{\delta}{6 A_{\mu}^{b}(x, t)} .
\end{gathered}
$$

It follows from eqs. (1.1) and (1.4) that

$$
\langle\dot{\Phi}[A(\cdot, t)]\rangle_{\eta}=\left\langle\int d ^ { t } x \left[ D_{\nu}^{a b} F_{\nu \mu}^{b}(x, t)+\eta_{\mu}^{a}(x, t)\left|\frac{\delta \Phi[A(\cdot, t)]}{\delta A_{\mu}^{a}(x, t)}\right\rangle,\right.\right.
$$

which contains no explicit dependence on the Zwanziger function [42]. This argument certainly shows that $\Phi$ picks up no $Z$-dependence during the first instant of Gfth-time evolution, but, unfortunately, one must be suspicious of further, regulator dependent, $Z$-dependence lusking in the $\eta$-term of eq. (1.6). Further investigation of this term leads one closer to Zwanziger's original Fokker-Planck language, which we now briefly review.

In the unregulated case, Zwanziger [10] analyzes the Fokker-Planck equaisation, tince the realting action for the noise is bottomless. 
tion, whose formal solution is

$$
\rho_{r p}[A ; t]=e^{-\left(t-t_{0}\right)\left(L+L_{s}\right)} \rho_{p p}\left[A ; t_{0}\right]
$$

where

$$
L=\int d^{4} x \frac{\delta}{\delta A_{\mu}^{a}(x)}\left[D_{\nu}^{a b} F_{\nu \mu}^{s}(x)-\frac{\delta}{\delta A_{\mu}^{a}(x)}\right], \quad L_{s}=\int d^{4} x \frac{\delta}{\delta A_{\mu}^{a}(x)} D_{\mu}^{a b} Z^{b}(x),
$$

and

$$
\rho_{x p}\left[A ; t_{0}\right]=\delta\left[A(\cdot)-A\left(\cdot, t_{0}\right)\right]
$$

Here functional derivatives act on everything to the right, and $A_{\mu}^{a}\left(x, t_{0}\right)$ is the initial condition of the Langevin equation. The observables are then computed from eqs. (1.7) and (1.9) as

$$
\begin{aligned}
\langle\Phi[A ; t]\rangle & =\int D A \Phi[A(\cdot)] \rho_{\rho p}[A ; t] \\
& =e^{-\left(l-t_{0}\right)\left(L^{+}+L^{+}\right)} \Phi\left[A(\cdot) \|_{0},\right.
\end{aligned}
$$

where

$$
L^{\dagger}=-\int d^{\dagger} x\left[D_{\nu}^{a b} F_{\nu \mu}^{\dagger}(x)+\frac{\delta}{\delta A_{\mu}^{a}(x)}\right] \frac{\delta}{\delta A_{\mu}^{a}(x)} ; \quad L_{\Sigma}^{\dagger}=\int d^{\dagger} x Z^{a}(x) G^{a}(x)
$$

are the dual operators and the subscript zero means to evaluate at $A(x)=$ $A\left(x, t_{0}\right)$. The $Z$ wanziger term, $L !$, which generates a gauge transformation, vanishes to all orders in $\left(t-t_{0}\right)$ when $\Phi$ is gauge invariant, since $L !$ is always applied to the gauge invariant objects $\left\{\left(L^{\dagger}\right)^{n} \Phi\right\}$. As we shall see below, this 
is not the case in the presence of stochastic regularization. 


\section{Incompatibility in Yang-Mills}

When the Langevin noise is fifth-time smeared, the system is non-Markovian, and the simple Folker-Planck formulation is lost. There remains a body of standard techniques, including the memory kernel analysis $[43]$, from which we draw here only the simplest aspects.

We begin by considering the Langevin equation (1.1) as a flow equation of the form $\dot{\vec{x}}=\vec{v}$. The density functional for the flow

$$
\rho[A(\cdot) ; t]=\delta\left[A(\cdot)-A^{\eta}(\cdot, t)\right]
$$

is related to the Fokker-Planck density as

$$
\rho_{m p}[A(\cdot) ; t]=\langle\rho[A(\cdot) ; t]\rangle
$$

and satisfies the equation of continuity $(\dot{\rho}+\nabla \cdot(\vec{v} \rho)=0)$

$$
\dot{\rho}\left[A(\cdot)_{i} t\right]+\left(L_{0}+L_{1}+L_{n}(t)\right) \rho[A(\cdot) ; t]=0
$$

Here

$$
L_{0}=\int d^{\top} x \frac{\delta}{\delta A_{\mu}^{a}(x)} D_{\nu}^{a b} F_{\nu \mu}^{d}(x), \quad L_{\eta}(t)=\int d^{\Lambda} x \eta_{\mu}^{a}(x, t) \frac{\delta}{\delta A_{\mu}^{a}(x)}
$$

and $L$, is defined in eq. (1.8). As a result, we have a formal construction of the Fokker-Planck density

$$
\begin{aligned}
\left.\rho_{p p} \mid A(\cdot) ; t\right] & \left.=\left\langle T \exp \left[-\int_{t_{0}}^{t} d \tau \mid L_{0}+L_{p}+L_{\eta}(\tau)\right]\right]\right\rangle \rho_{p p}\left[A(\cdot) ; t_{0}\right] \\
& =e^{-\left(t-t_{0}\right)\left(L_{0}+L_{-}\right)}\left\langle T \exp \left[-\int_{t_{0}}^{t} d \tau \tilde{L}_{\eta}(\tau)\right]\right\rangle \rho_{p p}\left[A(\cdot) ; t_{0}\right]
\end{aligned}
$$


valid for any stochastic regularization ${ }^{3}$. Here $T$ denotes time ordering, while in eq. (2.5b) we have introduced an interaction picture with

$$
\tilde{L}_{\eta}(t) \equiv e^{\left(t-t_{0}\right)\left(L_{0}+L_{e}\right)} L_{\eta}(t) e^{-\left(t-t_{0}\right)\left(L_{0}+L_{e}\right)}
$$

The corresponding results for the observables are

$$
\begin{aligned}
\left\langle\Phi[A(\cdot, t)]_{\eta}\right. & =\left.\left\langle T^{*} \exp \left[-\int_{t_{0}}^{t} d \tau\left[L_{0}^{\dagger}+L_{s}^{\dagger}+L_{\eta}^{\dagger}(\tau)\right]\right]\right\rangle \Phi[A(\cdot)]\right|_{0} \\
& =\left\langle T^{*} \exp \left[-\int_{t_{0}}^{t} d \tau \tilde{L}_{\eta}^{\dagger}(\tau)\right]\right\rangle e^{-\left(t-t_{0}\right)\left(L_{0}^{\dagger}+L_{-}^{\dagger}\right) \Phi[A(\cdot)) \|_{0}} \\
& =\left\langle T^{*} \exp \left[-\int_{t_{0}}^{t} d \tau \tilde{L}_{\eta}^{\dagger}(\tau)\right]\right\rangle e^{-\left(t-s_{0}\right) L_{0}^{\dagger} \Phi[A(\cdot)) \|_{0}}
\end{aligned}
$$

where $T^{*}$ is anti-time ordering, $L_{\eta}^{\dagger}=-L_{\eta}$, and so on. In eq. (2.7c) we have assumed $\Phi$ to be gauge invariant and noted that $L_{0}^{\dagger}\left(L_{0}^{\dagger}\right)^{n} \Phi=0$, since $L_{0}^{\dagger}$ is gauge invariant. It is not difficult to check that our results, eqs. (2.5) and (2.7), reduce to eqs. (1.7) and (1.11) when the regulator is removed.

We are now in a position to see how stochastic regularization induces $Z$-dependence in expectations of gauge invariant quantities. The basic problem is that the $Z$ wanziger $L$ ! terms inside the $\eta$-average of eq. (2.7c) are "shielded" by factors of $L_{\eta}^{\dagger}$, so that $L_{z}^{\dagger}$ hits gauge covariant but not gauge

${ }^{9}$ We note in pasaing that stochnetic regularination is almost cortainly not an action regularieation echeme, sine the Fokkor-Planck equation is not complotely regularived: The operator $L_{0}$ bae an explicit $\delta^{D}(0)$ divergence in $D$-dimensions. Thin does not occur in $L_{0}^{\dagger}$, which operatee on the observables, but is a warning that there may be unfamiliar pitfalls in any renormalination acheme for stochatic regularietion. 
invariant objects, and fails to annihilate. To exhibit this clearly, we expand eq. (2.7c) thru second order in $L_{\eta}^{\dagger}$, using eq. (1.2), to obtain

$$
\begin{aligned}
\langle\Phi[A(\cdot, t)]\rangle= & e^{-\left.\left(t-t_{0}\right) L_{0}^{\dagger} \Phi\right|_{0}} \\
& +2 \int_{t_{0}}^{t} d t_{1} \int_{t_{0}}^{t_{1}} d t_{2} e^{-\left(t_{2}-t_{0}\right)\left(L_{0}^{t}+L_{t}^{t}\right)} K\left(t_{2}-t_{1}\right) e^{-\left.\left(t-t_{1}\right) L_{0}^{t} \Phi\right|_{0}}+\cdots
\end{aligned}
$$

The shielding effect, due to the functional derivative in $L_{\eta}^{\dagger}$, is clearly seen in the factor

$$
K\left(t_{2}-t_{1}\right) \equiv \alpha_{A}\left(t_{2}-t_{1}\right) \int \alpha^{\alpha} x \frac{\delta}{\delta A_{\mu}^{*}(x)} e^{\left(t_{1}-t_{1}\right)\left(L_{0}^{t}+L_{1}^{t}\right)} \frac{\delta}{\delta A_{\mu}^{0}(x)} .
$$

The $L \ddagger$ terms in $K\left(t_{2}-t_{1}\right)$ do not annihilate when applied to objects like $\left\{\delta\left(L_{0}^{\dagger}\right)^{n} \Phi / \delta A_{\mu}^{\circ}\right\}$, since these are only gauge covariant and not gauge invariant,

$$
L^{\dagger} \frac{\delta}{\delta A_{\mu}^{q}(x)}\left(L_{0}^{\dagger}\right)^{n} \Phi \neq 0
$$

Similar shielding is found to all orders in $L_{\eta}^{\dagger}$. It is also easy to see that the shielding disappears when we set $\alpha_{A}\left(t_{2}-t_{1}\right)=\delta\left(t_{2}-t_{1}\right)$, which results in a $Z$-independent form for eq. (2.8).

We have also studied the form (2.7a) as a power series in $\Delta=t-t_{0}$ (and the coupling g). With $\alpha_{A}(t)=\Lambda^{4}|t| \exp \left(-\Lambda^{2}|t| \mid / 2\right.$, the $Z$-dependence sets in at $O\left(g \Delta^{4}\right)$ for a generic $Z$ wanziger function $Z$, and at $O\left(g \Delta^{5}\right)$ for the popular choice $\alpha Z^{\circ}=\partial \cdot A^{\natural}$. It is not clear how seriously to take these results however, since the power series in $\Delta$ is afficted with "secular divergences", related to the ultraviolet behavior of the expansion $\exp \left[-p^{2} \Delta\right]=\sum\left(-p^{2} \Delta\right)^{n} / n !$. 
As a check on our finite time arguments above, we computed the (infinite time) one loop correction to the gauge field mass using $\alpha Z^{a}=\partial \cdot A^{a}$ and the techniques of Ref. [19]. We find an $\alpha$ dependent mass, which diverges as $A \rightarrow \infty$, in accord with the arguments above.

The diagrams contributing to the gluon mass are shown in Fig. VI-1. For simplicity, we only consider here the Zwanziger-Feynman gauge (where the gauge parameter $a=1$ ), so that the gluon Langevin Green functions are diagonal in tensor indices. Since similar computations have already been presented for the case of scalar electrodynamics in Chapter III, we will omit the details here.

In this case the sum of the contributions of the diagrams in Fig. IV-1a, which are equal, is

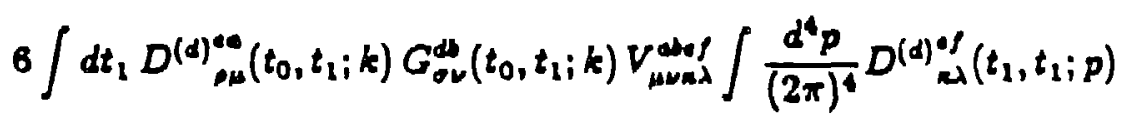

where

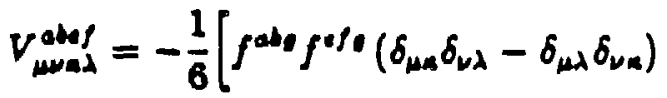

$$
\begin{aligned}
& +f^{\alpha \alpha \rho} f^{\phi / \rho}\left(\delta_{\mu \nu} \delta_{\mu \lambda}-\delta_{\mu \lambda} \delta_{\nu \alpha}\right) \\
& \left.+f^{a f \rho} f^{\alpha b \rho}\left(\delta_{\mu \alpha} \delta_{\nu \lambda}-\delta_{\mu \nu} \delta_{k \lambda}\right)\right]
\end{aligned}
$$

is the four-point Yang-Mills coupling. $G_{\mu \nu}^{a_{b}}\left(t_{1}, t_{2} ; k\right)$ is the Langevin Green function for the gluon, while

$$
D_{\mu \nu}^{(d)}=2 \delta_{\rho \sigma} \delta^{t d} \int d t_{3} \int d t_{1} G_{\mu \rho}^{a c} G_{\nu \rho,}^{b d} \alpha_{A}^{(d)}\left(t_{3}-t_{1}\right)
$$


and the regulator function $\alpha_{A}^{(d)}(\tau)$ is the given in eq. (2.9) of chapter III.

Truncating the external lega near $p=0$, performing the integrations and contracting the indices yields the zero momentum contribution to the vacuum polarization

$$
\Pi_{\mu \nu}^{(1)}(0)=-3 \frac{g^{2}}{16 \pi^{2}} N \delta^{\alpha \omega} \delta_{\mu \nu} \Lambda^{2}
$$

for $S U(N)$.

A similar, although more tedious computation for the diagrams in Fig. IV1b, yields the vacuum polarization contribution

$$
\Pi_{\mu \nu}^{(2)}(0)=2 \frac{g^{2}}{16 \pi^{2}} N \delta^{\omega \nu} \delta_{\mu \nu} A^{2}
$$

Since the sum of the two contributions does not vanish we find an explicit verification of the breakdown of gauge invariance caused by the incompatibility of Zwanziger's gauge fxing with stochastic regularization. We have also computed the one-loop vacuum polarization for arbitrary $\alpha$ and find similar results. 


\section{Incompatibility in Scalar Electrodynamics}

The incompatibility of Zwanziger's gauge fixing and stochastic regularization persists for any interacting gauge theory. In the case, e.g., of scalar electrodynamics, the gauge-fixed and regularized Langevin system is

$$
\begin{gathered}
\dot{A}_{\mu}=-\frac{\delta S}{\delta A_{\mu}}+\partial_{\mu} Z+\eta_{\mu} \\
\dot{\phi}=-\frac{\delta S}{\delta \phi^{*}}+i g \phi Z+\eta \\
\dot{\phi}^{*}=-\frac{\delta S}{\delta \phi}-i g \phi^{*} Z+\eta^{*},
\end{gathered}
$$

where

$$
\begin{gathered}
S=\int d^{4} x\left[\frac{1}{\gamma} F_{\mu \nu} F_{\mu \nu}+\left|\left(\partial_{\mu}-i g A_{\mu}\right) \phi\right|^{2}\right] \\
\left\langle\eta_{\mu}(x, t) \eta_{\nu}\left(x^{\prime}, t^{\prime}\right)\right\rangle_{\eta}=2 \alpha_{\Delta}\left(t-t^{\prime}\right) \delta_{\mu \nu} \delta^{4}\left(x-x^{\prime}\right) \\
\left\langle\eta^{-}(x, t) \eta\left(x^{\prime}, t^{\prime}\right)\right\rangle_{\eta}=2 \alpha_{\Delta}\left(t-t^{\prime}\right) \delta^{4}\left(x-x^{\prime}\right) .
\end{gathered}
$$

Following our reasoning above for Yang-Mills, we find analogous Z-dependence in gauge-invariant observables. If the observable in question, $\Phi[A \mid$, is a functional of only $A_{\mu}$, we find that the $Z$-dependence sets in at $O\left(g^{3}\right)$, two orders higher than Yang-Mills. The reason is that $\delta \Phi[A] / \delta A_{\mu}$ is still gauge invariant in the abelian case, so that two powers of the interaction are necessary to obtain shielding. As a consequence of this counting, a photon mass should not occur until two loops. In an explicit computation, we have confirmed that the photon picks up no mass at the one loop level. 


\section{Chapter V: Covariant Derivative Regularization}

\section{Regularised Langevin Systems for Gauge Theory}

For $S U(N)$ Yang-Mills theory in d-dimensions, we introduce the Markovian regularized Parisi-Wu [8] Langevin system

$$
\begin{aligned}
\dot{A_{\mu}^{a}}(x, t)= & -\frac{\delta S}{\delta A_{\mu}^{a}}(x, t)+D_{\mu}^{a b} Z^{b}(x, t)+\int(d y) R_{x y}^{a b}(\Delta) \eta_{\mu}^{b}(y, t) \\
& \left\langle\eta_{\mu}^{a}(x, t) \eta_{\nu}^{b}\left(y, t^{\prime}\right)\right\rangle_{\eta}=2 \delta^{a b} \delta_{\mu \nu} \delta\left(t-t^{\prime}\right) \delta^{d}(x-y)
\end{aligned}
$$

together with the usuai prescription for the computation of Euclidean Green functions

$$
\left\langleF \left[A ( \cdot ) | \rangle \equiv \operatorname { l i m } _ { t \rightarrow \infty } \left\langleF \left[ A(\cdot, t)|\rangle_{n}\right.\right.\right.\right.
$$

where $F[A]$ is any equal fifth-time functional of the gauge feld $A_{\mu}^{a}[\eta]$. Our notation here is

$$
\begin{gathered}
S=\frac{1}{4} \int(d x) F_{\mu \nu}^{a}(x) F_{\mu \nu}^{\omega}(x), \quad F_{\mu \nu}^{\omega} \equiv \partial_{\mu} A_{\nu}^{a}-\partial_{\nu} A_{\mu}^{a}-g f^{a \Delta d} A_{\mu}^{b} A_{\nu}^{c} \\
D_{\mu}^{\alpha \omega}=\delta^{\alpha \Delta} \partial_{\mu}+g f^{a \omega} A_{\mu \nu}^{a}, \quad(d x) \equiv d^{d x}
\end{gathered}
$$

and we have chosen to add to a Zwanziger gauge-fixing term $D_{\mu}^{\text {ab }} Z^{\bullet}$, which we will specify as $\alpha Z^{a}=\partial \cdot A^{a}$ for computational purposes. We will also check below, at the Schwinger-Dyson level, that gauge-invariant quantities do not depend on the gauge-fxing.

\footnotetext{
'Although 2wansiger'v gauge-fixing is deairable and natural in our scheme, other gaugeGinga, oucb an that of Ref. $[44 \mid$, may aloo be employed.
} 
The regulator $R_{x y}^{a b}(\Delta)$, which multiplies the white noise $\eta_{\mu}^{a}$ is a function of the covariant Laplacian

$$
\begin{aligned}
\Delta_{z y}^{a b} & \equiv \int(d z)\left(D_{\mu}\right)_{z x}^{a c}\left(D_{\mu}\right)_{s y}^{c b} \\
\left(D_{\mu}\right)_{x y}^{a b} & \equiv D_{\mu}^{a b}(x) \delta^{\alpha}(x-y)
\end{aligned}
$$

so that $R_{y z}^{\text {da }}(\Delta)=R_{x y}^{a b}(\Delta)$. In the absence of the gauge-fixing term, this construction, a gauge-covariant parallel transport of the noise, guarantees the gauge covariance of the regularized Langevin system under the local d-dimensional gauge transformation

$$
\begin{gathered}
\dot{A}_{\mu}^{\circ}(x, t) \rightarrow \Omega^{a b}(x) \dot{A}_{\mu}^{b}(x, t) \\
\eta_{\mu}^{\circ}(x, t) \rightarrow \Omega^{a b}(x) \eta_{\mu}^{b}(x, t) \\
R_{x y}^{a b}(\Delta) \rightarrow \Omega^{a \alpha^{\prime}}(x) \Omega^{\omega \omega^{\prime}}(y) R_{x y}^{a_{y}^{\prime \prime}}(\Delta)
\end{gathered}
$$

where $\Omega(x)$ e $S O\left(N^{2}-1\right)$ in in the adjoint representation of $S U(N)$.

Our scheme is therefore the natural generalization of the simple scalar prototype regularization studied in I [29]. As discussed in that reference, the hope for regularization lies in the fact that we have applied a gauge-covariant ultraviolet softening to the noise, which controls the quantum behavior of the theory. In particular, every closed loop contains at least one noise contraction, and therefore a certain amount of regularization.

To maintain the naive large cutoff limit, we must also require that $R(\Delta)$ approach unity as the cutoff $A$ goes to infinity. This leaves a very large class 
of possible regulator functions, from which we study here only the simplest set

$$
R^{(n)}=\left(1-\frac{\Delta}{d^{2}}\right)^{-n}, \quad n=1,2, \cdots
$$

although we mention that analytic forms such as the heat kernel regulator ${ }^{3}$ $R=\exp \left(\Delta / \Lambda^{2}\right)$ may be technically superior for nonperturbative analysis. The technical details of the important result that Yang-Mills theory in ddimensions is successfully regulated to all orders when we choose

$$
n \geq\left[\frac{d+1}{2}\right]
$$

where $[I]$ is the greatest integer less than or equal to $x$ can be found in Ref. [30]. When explicit examples are needed however, we shall concentrate on the four dimensional case, for which Yang-Mills is finite when $n \geq 2$.

The conventional first step in the weak coupling expansion of the Langevin equation (2.1) is the equivalent integral formulation

$$
\begin{aligned}
A_{\mu}^{a}(x, t)=\int_{-\infty}^{t} d t^{\prime}(d y) G_{\mu \nu}^{a b}\left(x-y, t-t^{\prime}\right) \\
\times\left[V_{\nu}^{b}\left(y, t^{\prime}\right)+\frac{1}{\alpha} Y_{\nu}^{b}\left(y, t^{\prime}\right)+\int(d z) R_{y=}^{b e} \eta_{\nu}^{e}\left(z, t^{\prime}\right)\right]
\end{aligned}
$$

in which

$$
\begin{aligned}
G_{\mu \nu}^{a b}\left(x-y, t-t^{\prime}\right)=\delta^{a b} \theta\left(t-t^{\prime}\right) & \int(d p) e^{-i p \cdot(x-v)} \\
& \times\left[T_{\mu \nu} e^{-p^{2}\left(t-t^{\prime}\right)}+L_{\mu \nu} e^{-p^{2}\left(t-t^{\prime}\right) / a}\right]
\end{aligned}
$$

${ }^{3}$ The heat kernel regulator in discuseed in Ref. [34]. 
is the Langevin Green function, and $T_{\mu \nu}\left(L_{\mu \nu}\right)$ is the standard transverse (longitudinal) projection operator. Here we have defined the interaction terms

$$
\begin{gathered}
V_{\nu}^{b} \equiv-g f^{b e d}\left[\partial_{\sigma}\left(A_{\sigma}^{c} A_{\nu}^{d}\right)-\left(\partial_{\sigma} A_{\nu}^{c}\right) A_{\sigma}^{d}+\left(\partial_{\nu} A_{\sigma}^{c}\right) A_{\sigma}^{d}\right] \\
-g^{2} f^{b c d} f^{c / c} A_{\sigma}^{d} A_{\nu}^{d} A_{\sigma}^{d} \\
Y_{\nu}^{d} \equiv g f^{b c d} A_{\nu}^{d} \partial \cdot A^{\alpha}
\end{gathered}
$$

which come, respectively, from the action and the $Z$ wanziger term, and also employed the technical device of chooding $t_{0}=-\infty$, so that the system has equilibrated at any faite fifth-time.

Before attempting an iterative weak coupling expansion of eq. (1.7), it is necessary to expand the regulator in powers of the coupling. As a first step in this expansion, we write

$$
\frac{\Delta_{\mathrm{zy}}}{\Delta^{2}}=\delta^{a b} \frac{\square_{\mathrm{zy}}}{\Delta^{2}}+g\left(\Gamma_{1}\right)_{z y}^{a b}+g^{2}\left(\Gamma_{2}\right)_{z y}^{a b}
$$

where the regulator "vertices" $\Gamma_{1}$ and $\Gamma_{2}$ are defined as

$$
\begin{gathered}
\left(\Gamma_{1}\right)_{s \nu}^{\alpha b} \equiv f^{a b c}\left(\partial_{\mu}^{z} A_{\mu}^{a}(x)+A_{\mu}^{c}(x) \partial_{\mu}^{a}\right) \delta^{d}(x-y) / A^{2} \\
\left(\Gamma_{2}\right)_{z \nu}^{a d} \equiv f^{\infty 00} f^{c l v e} A_{\mu}^{g}(x) A_{\mu}^{a}(x) \delta^{d}(x-y) / A^{2} .
\end{gathered}
$$

In (2.11) the derivatives $\partial_{\mu}^{x}$ act on everything to the right. In an obvious matrix notation, we may then expand $R^{(1)}$ to all orders as

$$
R^{(1)}(\Delta)=\frac{1}{1-\Delta / A^{2}}=\sum_{n=0}^{\infty}\left[R_{0}\left(g \Gamma_{1}+g^{2} \Gamma_{2}\right)\right]^{m} R_{0}
$$


where

$$
\left(R_{0}\right)_{s y}^{a b} \equiv \delta^{a b}\left[\left(1-\square / A^{2}\right)^{-1}\right]_{z v}
$$

Similarly, using the fact that $R^{(n)}=\left(R^{(1)}\right)^{n}$, we may expand $R^{(n)}$ to arbitrarily high order as

$$
\begin{aligned}
& R^{(n)}=R_{0}^{n}+\sum_{k, l=1}^{n} \delta_{k+\ell_{1} n+1} R_{0}^{h}\left(g \Gamma_{1}+g^{2} \Gamma_{2}\right) R_{0}^{\ell} \\
& +\sum_{l, l=m=1}^{n} \delta_{h+\ell+m, n+2} R_{0}^{k} g \Gamma_{1} R_{0}^{\ell} g \Gamma_{1} R_{0}^{m}+0\left(g^{3}\right) .
\end{aligned}
$$

Further discussion of these expansions, as well as a tree-graphical representation of the expansions to all orders is given in Appendix B of Ref. [30].

Having expanded the regulator to any desired order, as above, the integral equation (2.7) may be iterated in a conventional [8] fashion to expand the Langevin field

$$
A_{\mu}[\eta]=\sum_{m=0}^{\infty} g^{m} A_{\mu}^{(m)}[\eta]
$$

to arbitrarily high order as well. As an example, the result for the regulator $R^{(2)}(\Delta)$, which is minimal for the Langevin syotem $(2.1)$ in $d=4$ dimensions, takes the form,

$$
\begin{aligned}
A^{(0) a}\left(x_{1}, t_{1}\right) & =\int_{2} G_{u \nu}^{a b}\left(x_{1}-x_{2}, t_{1}-t_{2}\right)\left(R_{0}^{2} \eta\right)_{v}^{b}\left(x_{2}, t_{2}\right) \\
g A^{(1)^{a}}\left(x_{1}, t_{1}\right) & =\int_{2} G_{u \nu}^{a b}\left(x_{1}-x_{2}, t_{1}-t_{2}\right)\left\{g V^{(0) b}\left(x_{2}, t_{2}\right)+g \frac{1}{\alpha} Y^{(0)^{b}}\left(x_{2}, t_{3}\right)\right. \\
& \left.+g \int\left(d x_{3}\right)\left[R_{0}^{2} \Gamma_{1}^{(0)} R_{0}+R_{0} \Gamma_{1}^{(0)} R_{0}^{2}\right]_{\nu o}^{b c}\left(x_{2}, x_{3} ; t_{2}\right) \eta_{0}^{c}\left(x_{3}, t_{2}\right)\right\}
\end{aligned}
$$




$$
\begin{aligned}
g^{2} A_{\mu}^{(2) a}\left(x_{1}, t_{1}\right) & =\int_{2} G_{\mu \nu}^{a b}\left(x_{1}-x_{2}, t_{1}-t_{2}\right)\left\{g^{2} V_{\nu}^{(1) b}\left(x_{2}, t_{2}\right)+g^{2} \frac{1}{\alpha} Y^{(1) b}\left(x_{2}, t_{2}\right)\right. \\
& +g^{2} \int\left(d x_{3}\right)\left[R_{0}^{2} \Gamma_{1}^{(1)} R_{0}+R_{0} \Gamma_{1}^{(1)} R_{0}^{2}+R_{0} \Gamma_{2}^{(0)} R_{0}^{2}+R_{0}^{2} \Gamma_{2}^{(0)} R_{0}\right. \\
& +R_{0}^{2} \Gamma_{1}^{(0)} R_{0} \Gamma_{1}^{(0)} R_{0}+R_{0} \Gamma_{1}^{(0)} R_{0}^{2} \Gamma_{1}^{(0)} R_{0} \\
& \left.\left.+R_{0} \Gamma_{1}^{(0)} R_{0} \Gamma_{1}^{(0)} R_{0}^{2}\right]_{\omega 0}^{b c}\left(x_{2}, x_{3} ; t_{2}\right) \eta_{0}^{c}\left(x_{3}, t_{2}\right)\right\}
\end{aligned}
$$

where

$$
\begin{array}{rlrl}
V^{(m)} & \equiv V\left(A^{(m)}\right), & Y^{(m)} \equiv V\left(A^{(m)}\right), \\
\Gamma_{1}^{(m)} \equiv \Gamma_{1}\left(A^{(m)}\right), & \Gamma_{2}^{(m)} \equiv \Gamma_{2}\left(A^{(m)}\right), \\
\int_{1} \equiv \int\left(d x_{1}\right) d t_{1} .
\end{array}
$$

More useful at arbitrary order is the equivalent description in terms of Langevin tree graphs, which are easily derived from eqs. (1.7) or (1.16), using the tree-graphical expansions of the regulator given in Appendix B of Ref. $\{30\}$. The Langevin tree graphs in the case $n=2$ through $O\left(g^{2}\right)$ are ohown in Fig. V-1. These diagrams may be construeted to all orders using the Langevin tree ruled given in Fig. V-2.

We call attention to some salient features of the $n=2$ Langevin trees and their rules:

1. Vertices: In addition to the two ordinary Yang-Mills vertices (which 
include the $\mathrm{Z}$ wanziger contributions), there are exactly two extra regulator vertices proportional to $A^{-2}$, which represent $\Gamma_{1}$ and $\Gamma_{2}$ discussed above. To help distinguish these regulator vertices from the ordinary Yang-Mills vertices, we have placed a dot at the center of each regulator vertex.

2. Propagators: The wavy lines are Langevin Green functions, which are represented by directed lines because they are retarded in fifthtime. We will refer to these as field lines. Additionally, there are two types of regulator propagators, being single $\left(R_{0}\right)$ and double $\left(R_{0}^{2}\right)$ solid lines, which count the number of free regulator factors $R_{0}\left(p^{2}\right)=$ $\left(1+p^{2} / \Delta^{2}\right)^{-1}$, where $\int(d p) e^{i p \cdot(z-v)} R_{0}\left(p^{2}\right)=\left(R_{0}\right)_{s y}$. We remark that the regulator propagators are irstantaneous in fifth-time, as seen explicitly in eq. (1.16). This reflects the Markovian property of our regularization.

Note also that the Langevin trees eontain regulator strings of regulator propagators. These strings, which correspond to the regulator expansion discussed in Appendix B of Ref. [30), consist of sets of single or double regulator line propagators laid end to end at regulator vertices. In addition to any number of single-line regulator propagators, each such regulator string contains exactly one double-line regulator propagator, as seen explicitly in eq. (1.16). This is a characteristic of the $R^{(2)}$ regulator function. Whatever 
its length, of course, the entire string occurs at the same instant of fifth-time.

The tree rule regulator vertices for $R^{(n)}$, are the same as shown for $n=2$ in Fig. V-2, while a regulator propagator in an $R^{(n)}$ string may contain up to the nth power of $R_{0}$. Further details are given in Appendix B of Ref. [30].

The Langevin diagrams themselves are finally constructed by contracting the tree diagrams with the rule (2.1b) in the prescription (2.1c). Following Refs. [27] and [29], we place a cross at each such noise contraction. In general, therefore, a crose is the joining of two regulator strings of arbitrary length, and the entire contracted pair of strings is instantaneous.

As the simplest example, we mention the zeroth order two-point function shown in Fig. V-3. This diagram contains two Langevin Green functions $G_{\mu \nu}^{a b}$ and two powers of $R^{(2)}$ in the combination

$$
\begin{aligned}
& D_{\mu \omega}^{\omega \omega}\left(t_{1}, t_{2} ; p\right) \equiv 2 \int_{-\infty}^{t_{1}} d t_{3} \int_{-\infty}^{t_{2}} d t_{4} G_{\mu \infty}^{\infty a}\left(t_{1}-t_{3} ; p\right) G_{\nu \sigma}^{t_{\infty}}\left(t_{2}-t_{4} ; p\right) \delta\left(t_{3}-t_{4}\right) R_{0}^{4}\left(p^{2}\right) \\
& =\delta^{\alpha \omega}\left[T_{\mu \nu} e^{-p^{2}\left|t_{1}-t_{2}\right|}+\alpha L_{\mu \nu} e^{-p^{2}\left|t_{1}-t_{2}\right| / \alpha}\right] \frac{A^{8}}{p^{2}\left(p^{2}+A^{2}\right)^{4}} .
\end{aligned}
$$

The result for the regularized free gluon propagator is therefore

$$
\begin{aligned}
\left\langle A_{\mu}^{a}\left(x_{1}\right) A_{\nu}^{b}\left(x_{2}\right)\right\rangle & =\int(d p) e^{-i p \cdot\left(x_{1}-s_{2}\right)} D_{\mu \nu}^{\alpha d}\left(t_{1}, t_{1} ; p\right) \\
& =\delta^{\alpha b} \int(d p) e^{-i p \cdot\left(x_{1}-x_{2}\right)}\left[T_{\mu \nu}+\alpha L_{\mu \nu}\right] \frac{A^{8}}{p^{2}\left(p^{2}+A^{2}\right)^{4}} .
\end{aligned}
$$

Other free Green functions are constructed according to the usual Wick expansion in terms of the result (1.19). 
In general, any line in a Langevin diagram of the form of Fig. V-3 is represented by $D_{\mu \nu}^{a b}\left(t_{1}, t_{2} ; p\right)$. We shall call this simplest type of contraction (with no regulator vertices) a simple contraction, since it was the only possible contraction in the scalar prototype [29].

In the next section, we apply these Langevin rules to the computation of the one-loop gluon mass. 


\section{Vanishing Gluon Mass}

As an introduction to the loop structure of our regularization scheme, and also as an explicit check of gauge-invariance, we will verify in this section that the QCD, gluon mass remains zero at the one-loop level, with the regulator $R^{(2)}$. At the end of the computation, we will also discuss the failure of $R^{(1)}$ to regularize the theory.

As noted in our previous letter [27], there are forty-seven distinct Langevin graphs in the two-point function at order $g^{2}$, where diagrams trivially related by symmetry are not included in the count and will be included in the computations of this section via appropriate combinatoric factors. On closer examination, however, it is seen that only thirteen make non-zero contributions to the mase renormalization, while only two contribute to the wavefunction and gauge parameter (a) renormalizations.

We have found it convenient to group the forty-seven diagrams into four classes, of which only the first class contributes to the wavefunction and a-renormalizations, and only the first two classes contribute to the mass renormalization. The third class contributes only to the finite part of the vacuum polarization, which will not be considered in this paper, while the diagrams in the fourth class vanish identically.

Class_bs (three ordinary diagrams).

These diagrams, shown in Fig. V-4, are the ordinary non-vanishing Langevin 
diagrams that contain only (Zwanziger gauge-fixed) Yang-Mills vertices, no regulator vertices, and therefore only simple contractions. This class includes all the diagrams that contribute to the wavefunction and $\alpha$-renormalizations ( $p^{2} \ln A^{2}$ behavior), but, by themselves, these diagrams would yield a nonvanishing gluon mass (proportional to $A^{2}$ ), since they are regularized by what amounts to a naive gauge non-invariant cutoff, the free regulator $R_{0}$. Our scheme, of course, automatically provides other diagrams, with regulator vertices, to maintain the gauge-invariance.

Clase 2. (eleven eftra regulator contributions). The diagrams in this class (Fig. V.5) contain at least one $\Gamma_{1}$ or $\Gamma_{2}$ regulator vertex, and provide the additional gluon mass contributions needed to cancel the contribution of the ordinary graphs of class 1 . Since a regulator vertex carries an explicit factor of $A^{-2}$, the leading logarithmic behavior of any diagram with a regulator vertex is at most $\left(\ln \Lambda^{2}\right) / \Lambda^{2}$. It follows that diagrams with regulator vertices do not contribute to wave function or $\alpha$-renormalizations.

Clags 3 (twelve diagrams which are finite as $\Lambda \rightarrow \infty$ ). These diagrams, shown in Fig. V-6, also contain regulator vertices, but contribute only to the finite part of the vacuum polarization. The absence of contributions to mass renormalization follows on dimensional grounds, looking only at external legs: These diagrams never have two $G$ 's contracting into a $D$ (as in eq. (1.18)) on an external line. This means that they lack a factor of $p^{-2}$ relative to the diagrams of class 2 . After truncation, therefore, these 
diagrams contribute nothing to $\Pi_{\mu \nu}(0)$. Finiteness as $\Lambda \rightarrow \infty$ then follows, since the diagrams contain regulator vertices, and so cannot contribute to the wave function or $\alpha$-renormalizations.

Class 4 (twenty-one diagrams which vanish identically). This final class of diagrams is shown in Fig. V-7. The tadpole loops vanish as usual by $f^{\text {abe }}$ antisymmetry. The remaining diagrams vanish due to the (fifth-time) retarded property of the Langevin Green functions, which contribute a factor of $\theta\left(t_{1}-t_{2}\right) \theta\left(t_{2}-t_{1}\right)=0$ to each diagram.

As an explicit example, we evaluate the ordinary diagram V-4b, shown with all relevant indices in Fig. V-8. Using the Langevin tree rules of Fig. V. 2, we obtain for this diagram

$$
\begin{aligned}
& 8 \int d t_{1} \int d t_{2} \int(d k) G_{m}^{e a}\left(t_{0}, t_{1} ; p\right) D_{o u}^{d \omega}\left(t_{0}, t_{1} ; p\right) V_{\mu a d}^{a a f}(0,-k, k)
\end{aligned}
$$

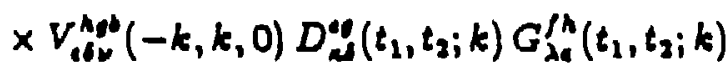

where $D_{\mu \nu}^{a t}\left(t_{0}, t_{1} ; p\right)$, corresponding to each simple contraction of the diagram, is defined in eq. (1.18). We have also neglected the dependence of the vertex factors on the external momentum p, since we are only interested here in the contribution to the gluon mass. After performing the fifth-time integrations, 
and some tensor algebra, we obtain the value for this diagram near $p=0$ as

$$
\begin{aligned}
& {\left[\delta^{\mathrm{ea}}\left(\frac{T_{\rho \mu}(p)}{p^{2}}+\frac{\alpha L_{\rho \mu}(p)}{p^{2}}\right)\right]} \\
& \times\left[2 g^{2} f^{0 f \cdot f^{t f o} \delta_{\mu \nu}}\left(\frac{5}{8}+\frac{3}{8} \alpha\right) \int(d k) \frac{A^{8}}{k^{2}\left(k^{2}+A^{2}\right)^{4}}\right] \\
& \times\left[\delta^{d \omega}\left(\frac{T_{\nu \sigma}(p)}{p^{2}}+\frac{\alpha L_{\nu \sigma}(p)}{p^{2}}\right)\right] .
\end{aligned}
$$

Truncation near $p=0$ is accomplished by removal of the two factors in brackets, reaulting in this diagram's contribution to the mass renormalization

$$
\begin{aligned}
\Pi_{\mu \nu}^{(1)}(0) & =\frac{1}{4}(5+3 \alpha) g^{2} \delta_{\mu \nu} N \delta^{\alpha \omega} \int(d k) \frac{\Delta^{\alpha}}{k^{2}\left(k^{2}+\Delta^{2}\right)^{4}} \\
& =\frac{1}{12}(5+3 \alpha) \frac{N g^{2}}{16 \pi^{2}} \delta_{\mu \nu} \delta^{\alpha b} \Delta^{2}
\end{aligned}
$$

for $S U(N)$. In units of $N g^{2} \delta_{\mu \nu} \delta^{a b} A^{2} /(4 \pi)^{2}$, this result is recorded with Fig. V$4 b$.

The contributing diagrams with regulator vertices are generally easier to evaluate. In the case, for example, of diagram V-5e, shown with all indices in Fig. V-9, we obtain for $p \rightarrow 0$

$$
\begin{aligned}
& 2 \int_{-\infty}^{t_{0}} d t_{1} G_{p \mu}^{\infty}\left(t_{0}, t_{1} ; p\right) R_{0}(p) \int(d k) G_{d}^{c k}\left(t_{1}, t_{1} ; k\right)
\end{aligned}
$$

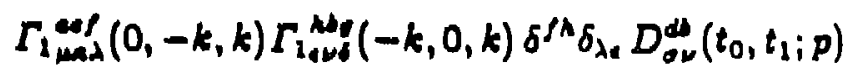

$$
\begin{aligned}
& =\left[\delta^{\mathrm{ec}}\left(\frac{T_{\mu \rho}(p)}{p^{2}}+\alpha \frac{L_{\mu \rho}(p)}{p^{2}}\right)\right] \\
& \times\left[-\frac{1}{2} g^{2} N \delta^{a b} \delta_{\mu \nu} \Lambda^{4} \int(d k) \frac{k^{2}}{\left(\Lambda^{2}+k^{2}\right)^{4}}\right] \\
& \times\left[\delta^{b d}\left(\frac{T_{\nu d}(p)}{p^{2}}+\frac{\alpha L_{\nu d}(p)}{p^{2}}\right)\right] \text {, }
\end{aligned}
$$


in which we have used

$$
G_{n \delta}^{e q}\left(t_{1}, t_{1}, k\right)=\frac{1}{2} \delta^{\operatorname{sg}} \delta_{\kappa \delta}
$$

Then, truncating as above, this diagram's contribution to the gluon mass is

$$
\Pi_{\mu \nu}^{(2)}{ }_{\mu \nu}^{\alpha}(0)=-\frac{1}{6} \frac{N g^{2}}{16 \pi^{2}} \delta^{a b} \delta_{\mu \nu} \Lambda^{2}
$$

as recorded under diagram V-5e.

The value of each of the remaining contributions in this computation is recorded, in units of $N g^{2} \delta_{\mu \nu} \delta^{a b} A^{3} /(4 \pi)^{3}$, under its diagram in Figs. V-4 and V-5. The reader may easily verify that the sum of all contributions is zero, so the gluon remains massless to this order.

The computation of the wavefunction and gauge parameter $(\alpha)$ renormalizations, which are controlled entirely by logarithmic contributions from the unual diagrame $V A b$ and $V A c$, has been given in Ref. [30]. We remark here only that the results are the same as that obtained by dimensional regularization of the Zwanziger gauge-fixed theory. This comes as no surprise, since these diagrams contain no regulator vertices.

We finally comment on the inadequacy of the regulator $R^{(1)}$ in four dimensions. In fact, the "ordinary" diagrams of class 1 are regularized by any member of the regulator family including $R^{(1)}$, but $n \geq 2$ is required for finiteness of certain class 2 diagrams, which contain regulator vertices. This is easily seen by looking bacx, for example, at diagram V-5e, and the 
corresponding eq. (2.4). With $R^{(2)}$, the loop integration of this diagram had the form $\int d^{4} k k^{2} R_{0}^{4}(k)<\infty$, whereas, with $R^{(1)}$, the double regulator line in the loop would be replaced by a single regulator line. This results in $\int d^{4} k k^{2} R_{0}^{3}(k)$, which is logarithmically divergent.

For $n \geq 2$ more powers of the regulator propagators will appear in these one-lcop diagrams, rendering the momentum integrals even more convergent, and the same vanishing gluon mass will be obtained. Finiteness of the regularized theory to all orders, when $n \geq 2$, has been discussed in Ref. [30]. 


\section{Chapter VI: Conclusions}

We discussed continuum regularization schemes for quantum field theory which were based upon the Langevin equation of Parisi and Wu.

The Breit, Gupta and Zaks stochastic regularization scheme [17] was analyzed for the case of scalar electrodynamics with the standard covariant gauge-fixing. Their scheme was shown to work, at least at the one-loop level, contrary to the ciaim in their original paper. (Other authors $[\mathbf{2 4 , 2 5}]$ have traced their results to diffculties associated with their use of a fermionic Langevin equation which was not manifestly gauge invariant.)

Although stochastic regularization may be viable perturbatively, difficulties arise which rule out its usefulness as a nonperturbative continuum regularization scheme. One problem is that the superficial quadratic divergences force a bottomless noise action. Another difficulty is that stochastic regularization by fifth-time smearing is incompatible with Zwanziger's gauge fixing.

Finally, a successful covariant derivative scheme is discussed, which avoids the difficulties encountered with the earlier stochastic regularization by fifthtime smearing. The regularized formulation is manifestly Lorentz invariant, gauge invariant, ghost free and finite to all orders. The gluon mass was shown to vanish at one loop All the technical advantages of a Markov process are retained, including closed form equilibrium equations, which are the 
Schwinger-Dyson equations, and Zwanziger's gauge fxing if desired. Further details can be found in Refs. $[29,30,31,32,34,35]$. We are hopeful that the scheme will lend itself to nonperturbative analysis. 


\section{References}

(1) B.W. Lee and J. Zinn-Juntin, Phys. Rov. DB, (1972) 3121

[2] A.A. Slạnov, TMP 33, (1977) 210

[3] L.D. Faddeev and A.A. Slavnov, Gauge Field: Introduction to Quentum Theory (BenjaminCummings, Reading 1980)

[4] L.D. Faddeev and V.N. Popov, Phys. Lett. B25 (1967) 30

[5] V.N. Gribov, Nuel Phys. B139 (1978) 1;

M.B. Halpers and J. Koplik, NacL. Phy. B132 (1978) 239

(6) M. Acorey and P.K. Mitter, Commun. Math. Phyo. 80 (1981) 43;

M. Acorey and P.K. Mitter, CERN-TR-3424/82

[7] I.M. Singer, Phyoica Scripta 24 (1981) 817

|8| G. Parini and Wu Yong-Shi, Sci. Sin. 24 (1981) 483

[9] P. Laagevia, C.R. Acad. Sei. Pari 146 (1908) 530;

M. Lax, Rav. Mod. Phyı. 38 (1986) 541;

N.G. Van Kampen, Stochartic Processes in Chemistry and Physics (North-Holland, Amuterd 2n, 1981)

(10) D. Zwansigor, Nuel. Phy. B192 (1981) 259;

E.G. Floraton, J. Diopoulon and D. Zwasiger, Nucl. Phye. B341 (1984) 221

[11] C. Bauliea and D. Zwanaiger, Nuel. Phya. B193 (1981) 103

D. Zwansigor, Phyo. Letto. 114B (1982) 337;

D. Zwansiger, Nuel. Phye. B200 (1982) 336

E. Seiler, MPI-PAE/PTb 20/84, publinhed in Schladming School (1984) 259, QCD161 (1984), 18 
[12] E.S. Chan and M.B. Halpern, Phyn. Rav. Dss (1986) 540;

A. Muños Sudupe and R.F. Alvarez-Eutrada, Phyc. Lett. 104B (1985) 102;

A. Muños Sudape and R.F. Alvares-Eatrada, Phys. Lett. 160B (1986) 186

(13) J. Alfaro and B. Sakita, Phyo. Lett. $121 B$ (1983) 339

[14] J. Greensite and M.B. Halpern, Nucl. Phye. B211 (1983) 343

(15) J. Greenoite and M.B. Halpern, NucL Phys. B242 (1984) 167

[16] A.J. Niemi and L.C.R. Wijewardhane, Ann. Phye. 140 (1982) 247

[17| J.D. Breit, S. Gupta and A. Zak, Nucl. Phys. B233 (1984) 61

[18] M. Namiki and Y. Yamencles, Eed. Jour. 7 (1984) 594

[19] 2. Bern, Nucl Phys. B251 (1905) 633

[20] J. Alfaro, Nucl. Phya. B28s (1985) 464

[21] M. Claudaon and M.B. Halpern, Phys. Rev. Ds1 (1985) 3310;

Z. Bern and Hoe Sun Chen, Nucl. Phyn. B206 (1986) 509;

Hue Sun Chan, LBL-20025;

Buv Sun Chen, LBL-31130

[22] H.W. Hamber and U.M. Heller, Phye. Rev. D20 (1984) 928;

G.G. Batroani, G.R. Kats, A.S. Kronfeld, G.P. Lepage, B. Svetitsky and K. G. Wileon, Phyc. Rav. D32 (1985) 2736

(23) T. Fatai, E. Natanako, I. Obba, K. Okano and Y. Yamanka, Prog. Theor. Phys. 69 (1983) 1600

P.H. Damgand and K. Trokon, Nuel. Phya. B2s8 (1984) 75;

R. Horaley and W. Schoenmaker, Pbyu. Rev. DS1 (1985) 822

|24| K. Ishikawa, Nucl. Phys. B241 (1984) 589

[25] U.G. Kaulfuns and U.B. Meinaner, TPR-85-17 
[26] Z. Bern and M.B. Halpern, Phys. Rev. Dss (1986) 1184

[27) Z. Bern, M.B. Halparn, L. Sadun and C. Taubes, Phys. Lett. 165B (1985) 151

[28] C.R. Doering, Phys. Rev. Lott. 55 (1985) 1657

[29] Z. Bern, M.B. Halpern, L. Sadun and C. Taubea, LBL-20646 UCB-PTH-85/52

[30] Z. Bern, M.B. Halpern, L. Sadun and C. Taubes, LBL-21117, UCB-PTH-86/4

(31) Z. Bern, M.B. Halpern, and L. Sadun, "Continuam Regularization of Quantum Field Theory III. The QCD, $\beta$-Function", in preparation

[32] Z. Bera, M.B. Ealpera and L. Sadun, CContinuam Regularisation of Quantum Field Theory IV. Further Aupecte of Renormalietion", in proparation

[33| R.T. Seloy, Aner. Math Soc. Proc. Symp. Pure Math 10 (1967) 288;

V.N. Romanor and A.S. Sehwars, Theor. Makh. Phys. \&1 (1979) 190.

[34] Z. Bern, M.B. Halpera and N.G. Kalive, LBL-21286, UCB-PTH-86/6

[35] Z. Bern, H.S. Chan and M.B. Halpern, in proparation

[36] B. Sakita, Proc. 7tb Jobne Hopkin Workehop (World Scienti6e, 1983)

[37| W. Grimus and B. HũfeL, 2. Phyı. C18 (1983) 129;

E. Egorian and S. Kalituin, Phye. Lott. 120B (1983) 320

[38] E. Gosui, CCNY-REP-84/3, 1984;

R. Kirachner, Phyo. Latt. 130B (1984) 180

[39] M.B. Eslpora (private communication)

[40] G. 't Hooft and M. Veltman, Nuel Phys. B4t (1972) 189

[11] C. Itsykson and J.B. Zuber, Quantum Field Theory, McGraw-Hill, New York, 1980 J.D. Bjorken and S.D. Drell, Roldtivistic Guantum Fields, McGraw-Hill, New York, 1965

[42] E. Floratom and J. Lliopoulon, Nuel. Pbys. B214 (1983) 392 
64

[43] E. Knobloch, Vintas in Astro. 24 (1980) 39 N.G Van Kampen, Physica 74 (1974) 239

[41 E. Hüfel and P.V. Landehoff, Nucl Phys. B260 (1985) 545 


\section{Figure Captions}

Fig. II-1: Perturbative Langevin expanaion of $\phi$ in $\frac{\lambda \phi^{\phi}}{4}$ theory.

Flg. II-2: Expansion of two point function in $\frac{\lambda \phi^{\natural}}{4 !}$ theory.

FIg. III-1: One Loop correction to the photon propagator in SED uning ordinary Feynman diagrams.

Flg. II-2: One Loop correction to the photon propagator in SED uring Langevin diagrams.

Flg. III-3: Complete three point function in acalar electrodynamics.

Fig. III-4: Complete ecalar propagator in ecalar electrodynamics.

Fif. IV-1: Disgrems that contribute to the gluon maes.

Fig. V-1: Laggevin tren diagrame through $O\left(g^{2}\right)$.

Fig. V-2: Langevin tree rules aging $R^{(2)}$.

FIg. V-s: A simple contraction.

Flg. V-4: "Ordinary" non-vaniehing Langevin diagrams.

Fig. V-6: Diagrame with regulator vertices that ateo contribute to gluon maes.

Fig. V-6: Diagrams with regulator vertice, which are finite as $A \rightarrow \infty$.

Fig. V.T: Diagram that vanish identically.

Flg. V-8: Diagram 3.16 with relevant indices.

Pig. V-9: Diagram 3.20 with relevant indicet. 


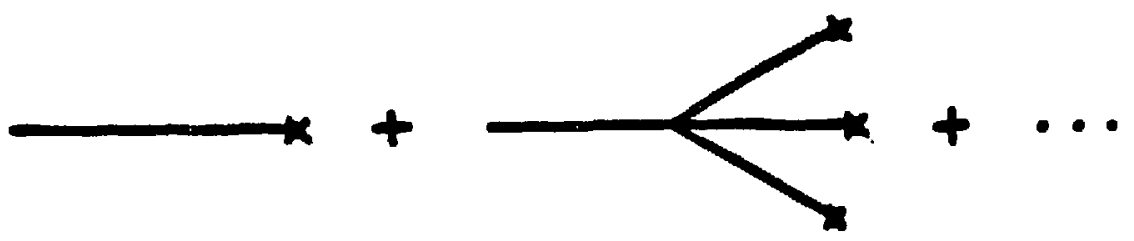

Fig. II-1

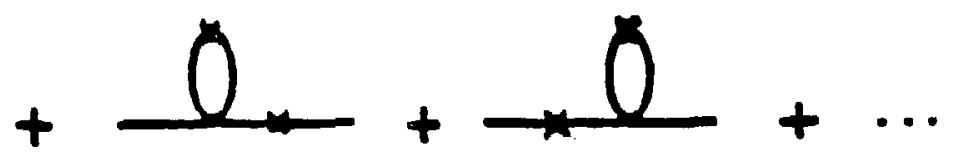

Fig. II-2

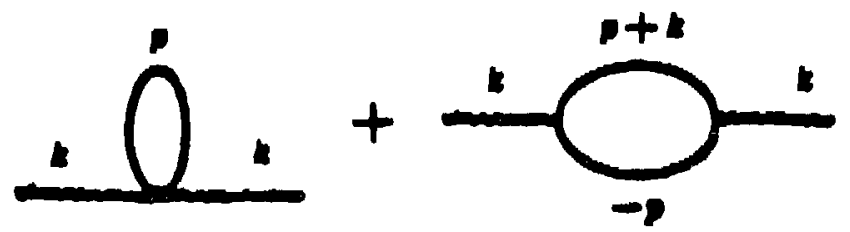

Fig. III-1 

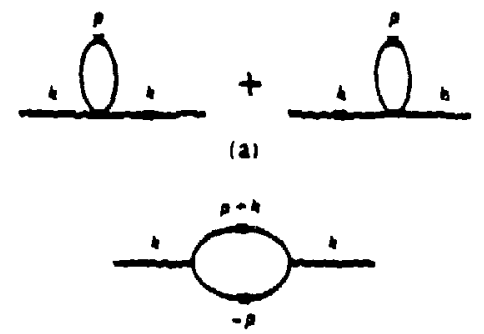

(b)

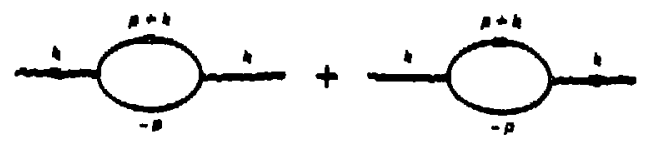

(c)

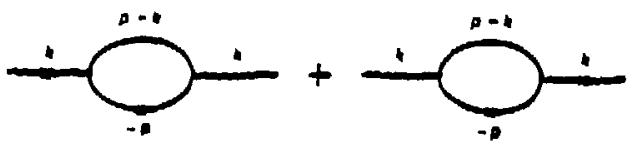

(d)

Fig. III-2

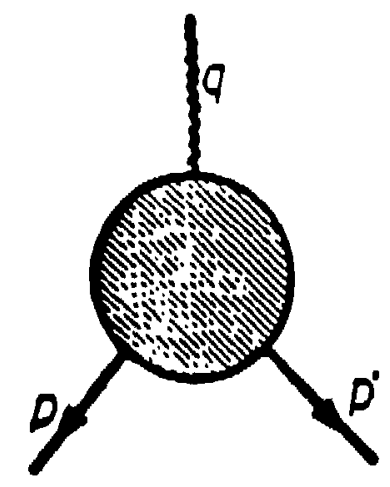

Fig. III-3

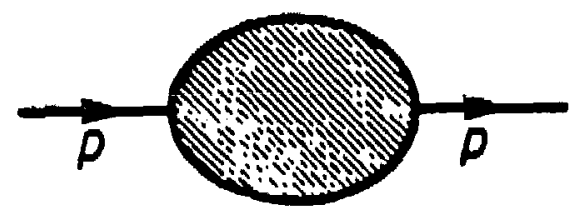

Fig. III-4 

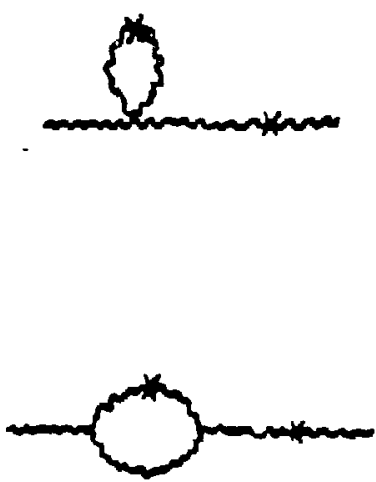

$+$

(b)

(a)

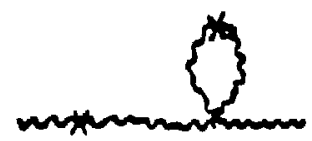

Fig. IV-1

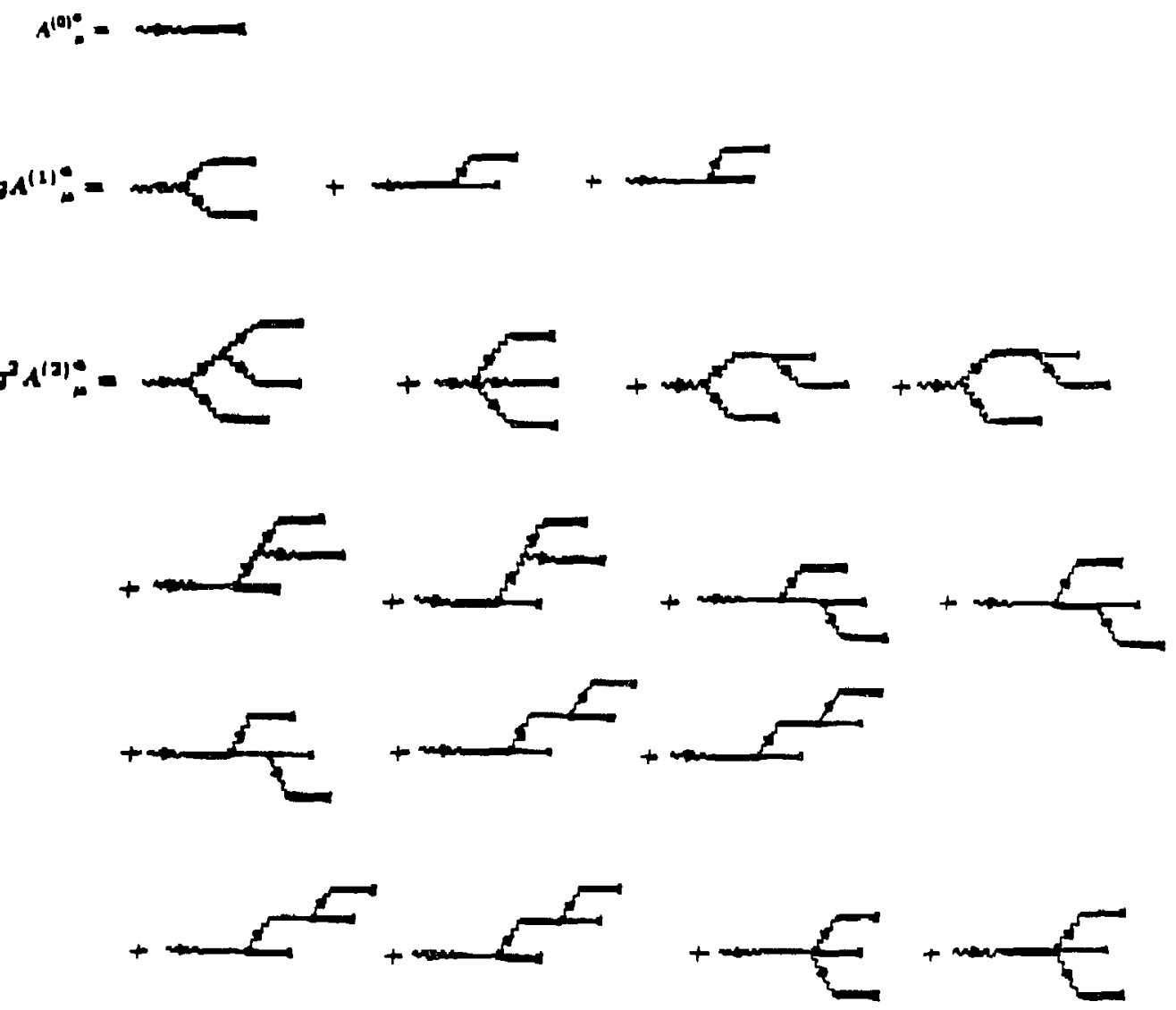

Fig. V-1 


\section{gaventas:}

$$
\begin{aligned}
& 4 \underset{-12}{-\infty}=G_{2}\left(t_{1}-l_{2}, p\right)
\end{aligned}
$$

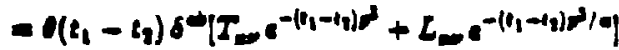

$$
\begin{aligned}
& t_{1} \div-10 t_{2}=\delta+6\left(t_{1}-t_{1}\right) \frac{1}{1+p^{2} / A^{2}}
\end{aligned}
$$

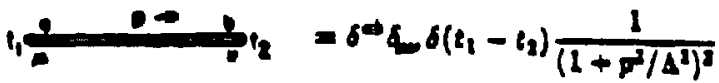

\section{Ginet}
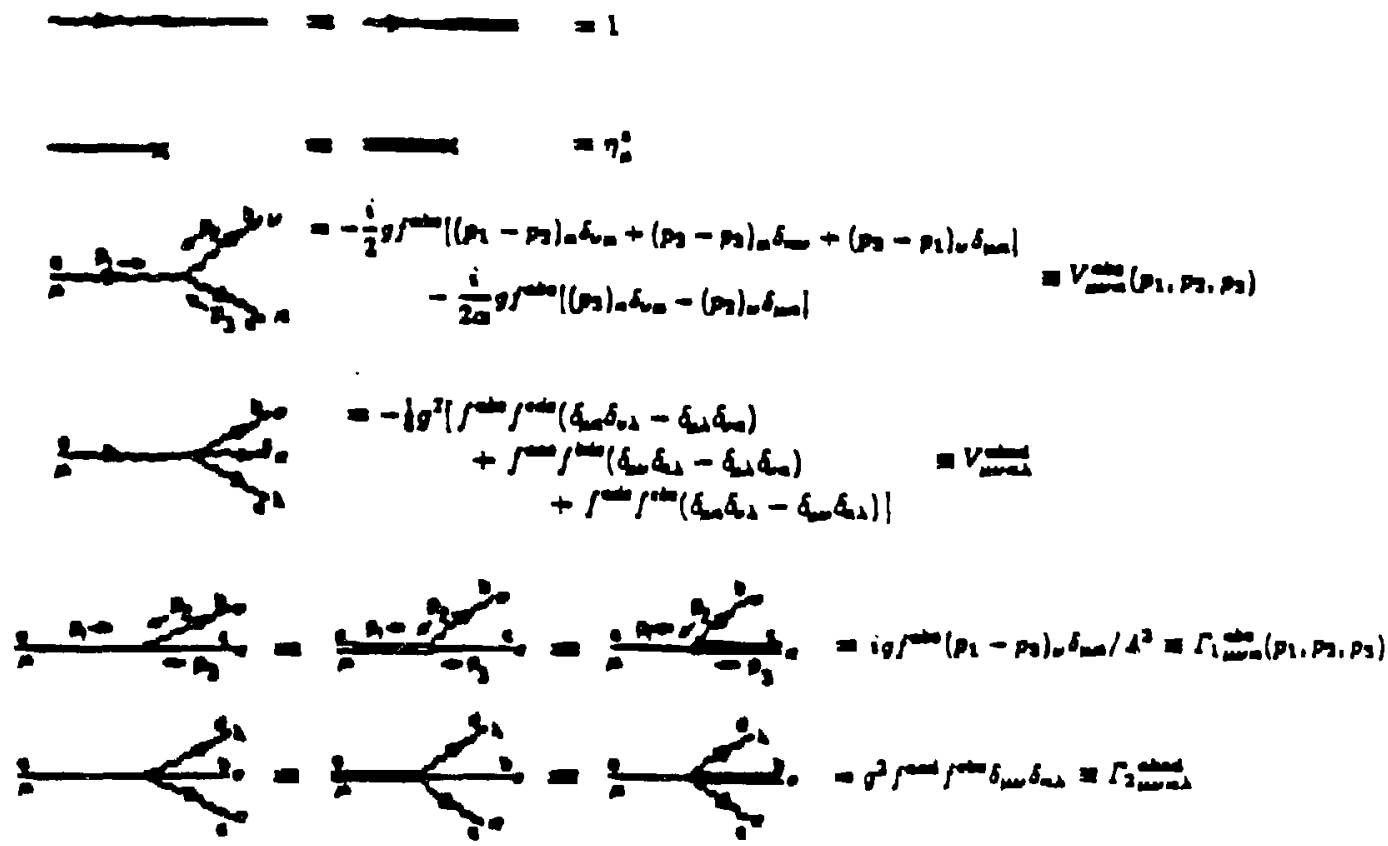

Fig. V-2

Fig. V-3 


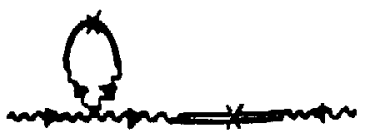

(a): $-(3+a) / 4$

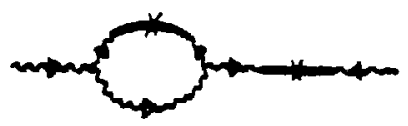

(b): $(5+3 a) / 12$

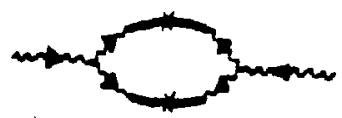

(c): 0

Fig. V-4

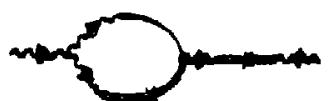

(c): $1 / 0$

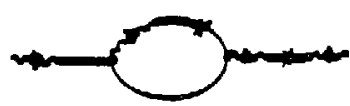

(e): $-1 / 6$

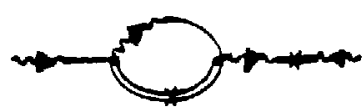

(i): $-1 / 24$

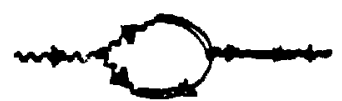

(b): 1/6

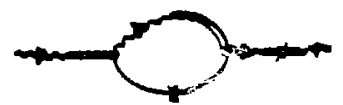

(l): $-1 / 5$

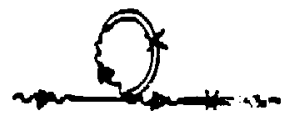

(j): $1 / 6$

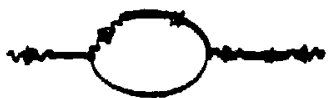

(c): $-1 / 24$

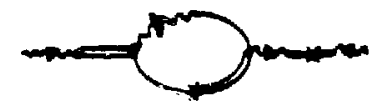

$(5):-1 / 6$

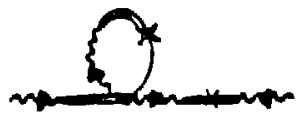

(k): 1/2

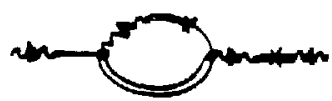

(d): $-1 / 24$

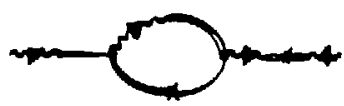

(b): $-1 / 24$

Fig. V.:
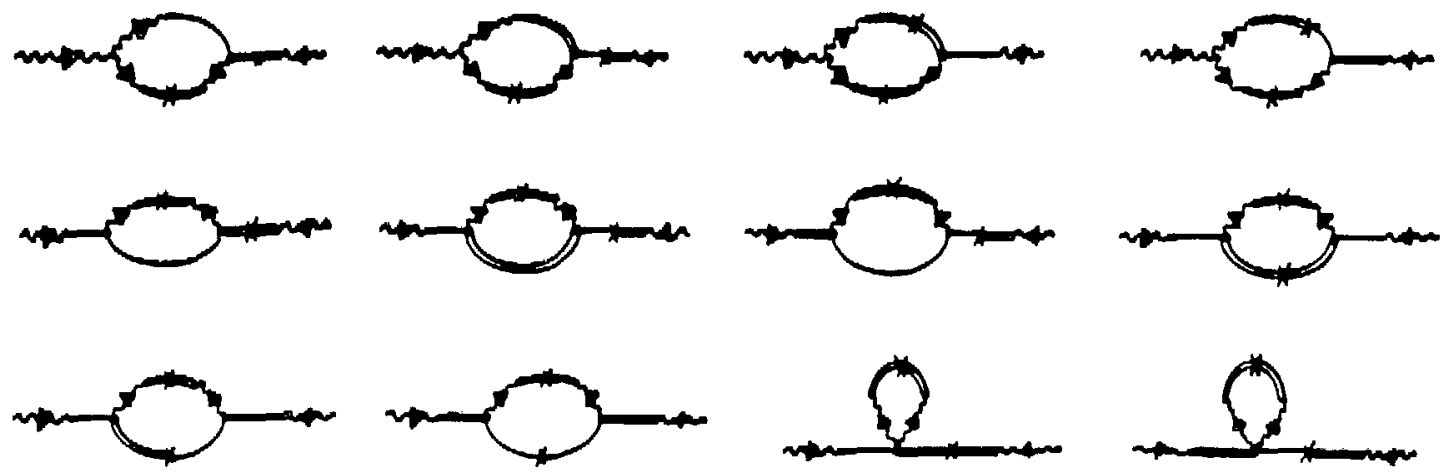

Fig. V-6 

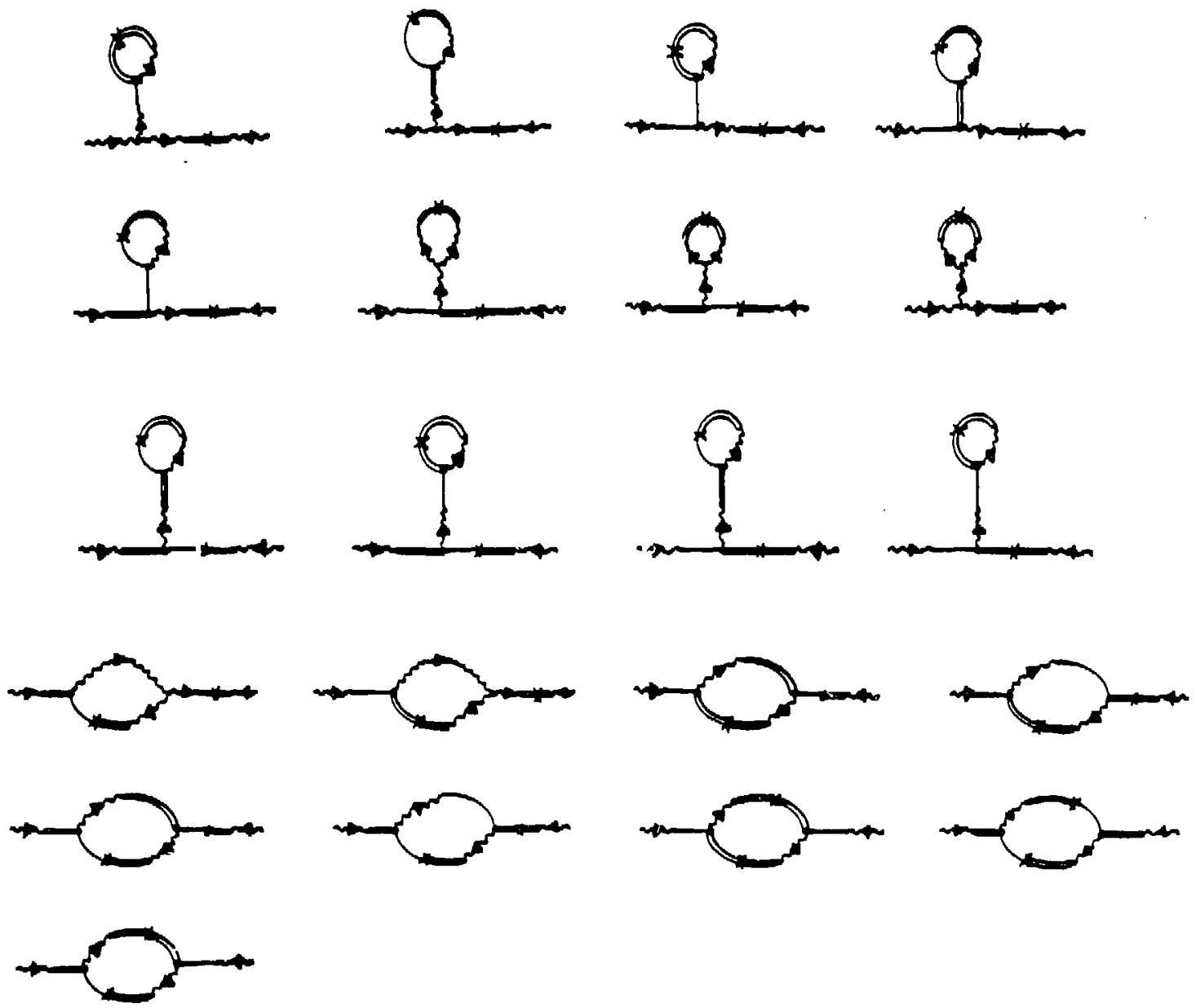

Fig. V-7

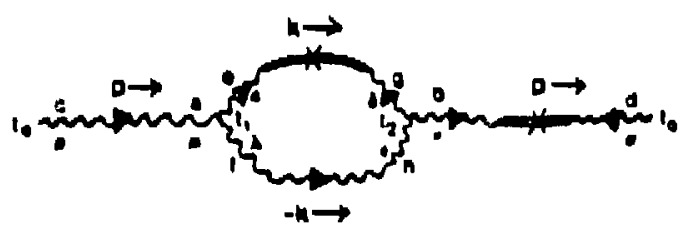

Fig. V-8

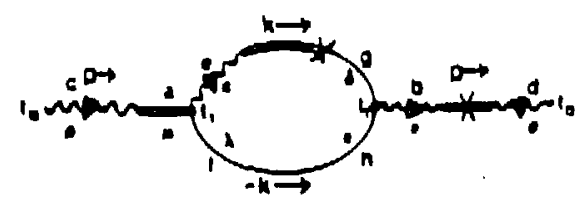

Fig. V-9 